\title{
Traumatic Brain Injury Preserves Firing Rates But Disrupts Laminar Oscillatory Coupling and Neuronal Entrainment in Hippocampal CA1
}

\author{
DPaul F. Koch, ${ }^{1}$ Carlo Cottone, ${ }^{1}$ Christopher D. Adam, ${ }^{1}$ 'Alexandra V. Ulyanova, ${ }^{1}$ Robin J. Russo, ${ }^{1}$ \\ Maura T. Weber, ${ }^{1}$ John D. Arena, ${ }^{1}$ Victoria E. Johnson, ${ }^{1}$ and ${ }^{1}$ John A. Wolf ${ }^{1,2}$
}

https://doi.org/10.1523/ENEURO.0495-19.2020

${ }^{1}$ Center for Brain Injury and Repair, Department of Neurosurgery, University of Pennsylvania, Philadelphia, Pennsylvania 19104 and ${ }^{2}$ Corporal Michael J. Crescenz Veterans Affairs Medical Center, Philadelphia, Pennsylvania 19104

\begin{abstract}
While hippocampal-dependent learning and memory are particularly vulnerable to traumatic brain injury (TBI), the functional status of individual hippocampal neurons and their interactions with oscillations are unknown following injury. Using the most common rodent TBI model and laminar recordings in CA1, we found a significant reduction in oscillatory input into the radiatum layer of CA1 after TBI. Surprisingly, CA1 neurons maintained normal firing rates despite attenuated input, but did not maintain appropriate synchronization with this oscillatory input or with local high-frequency oscillations. Normal synchronization between these coordinating oscillations was also impaired. Simultaneous recordings of medial septal neurons known to participate in theta oscillations revealed increased GABAergic/glutamatergic firing rates postinjury under anesthesia, potentially because of a loss of modulating feedback from the hippocampus. These results suggest that TBI leads to a profound disruption of connectivity and oscillatory interactions, potentially disrupting the timing of CA1 neuronal ensembles that underlie aspects of learning and memory.
\end{abstract}

Key words: hippocampus; medial septum; oscillations rodents; synchronization; TBI

\section{Significance Statement}

The hippocampus and its connections have been hypothesized to be particularly vulnerable to traumatic brain injury (TBI) and therefore are implicated in post-TBI memory impairments. However, it remains an open question whether loss of neurons, their activity, or an encoding disruption underlies such deficits, and whether the remaining neurons are capable of recovering the function of these networks. Using laminar silicon probes spanning the entire CA1 subregion, this study provides the first in vivo evidence that hippocampal CA1 single unit activity post-TBI can maintain a normal firing rate despite significantly attenuated, layerspecific loss of input. They cannot, however, maintain normal synchronization to the dominant oscillations within the hippocampus, a critical component of hippocampal memory encoding and decoding mechanisms.

\section{Introduction}

Learning and memory impairments are a common and debilitating consequence of traumatic brain injury (TBI) for which there are few effective treatments, presenting a

Received November 27, 2019; accepted July 19, 2020; First published July 31, 2020.

The authors declare no competing financial interests. significant burden to society (Capruso and Levin, 1992; Monti et al., 2013; Maas et al., 2017; Wilson et al., 2017). While the neuropathologies of $\mathrm{TBI}$ are diverse and

Author contributions: P.F.K. and J.A.W. designed research; P.F.K., R.J.R., and M.T.W. performed research; P.F.K., C.C., C.D.A., A.V.U., R.J.R., M.T.W., J.D.A., and V.E.J. analyzed data; P.F.K., A.V.U., V.E.J., and J.A.W. wrote the paper. 
heterogeneous, the hippocampus $(\mathrm{HC})$ and its connections have been reported as highly vulnerable to traumatic injury both in humans and multiple experimental TBI models (Smith et al., 1991, 1997; Hicks et al., 1993; Blumbergs et al., 1995; Toth et al., 1997; Grady et al., 2003). Moreover, impairment of learning and memory tasks following experimental TBI has long been observed in association with hippocampal pathology and changes in hippocampal circuitry, largely through ex vivo investigations in slice preparations (Reeves et al., 1997; Howard et al., 2007; Norris and Scheff, 2009; Almeida-Suhett et al., 2015; Wolf et al., 2017). Comparatively little evidence has been reported describing alterations in hippocampal network-level neuronal activity patterns in the intact brain that are thought to support the ability to encode and decode information. These disruptions may underlie hippocampal-dependent memory deficits repeatedly observed after experimental TBI (Smith et al., 1997).

Synchronized hippocampal synaptic activity can be observed as distinct, organized oscillations within the local field potential (LFP), each with unique signatures that include preferred frequency bands as well as patterns of power and phase at different depths within the anatomic organization of the hippocampal layers. These oscillations are crucial for hippocampal-dependent memory performance, and include theta, gamma, and sharp wave-associated ripple (SPW-R) oscillations (O'Keefe and Recce, 1993; Foster and Wilson, 2006; Lisman and Buzsáki, 2008; Buzsáki, 2015). Each of these represent different patterns of coordinated synaptic input and can drive the firing patterns of local hippocampal pyramidal cells and interneurons depending on the current network and behavioral state. The relationship between coordinated synaptic input into the hippocampus (observed as LFP oscillations and waves), and the timing of local neuronal activity is an important component of information encoding, recall, and, potentially, consolidation (Carr et al., 2011; Buzsáki and Moser, 2013). Whether coordination of synaptic inputs at the hippocampal network level or neuronal firing patterns driven by these inputs become disrupted after TBI is unknown.

Several reports from animal models have described a loss of hippocampal theta power after TBI that correlated

This research was supported by The Department of Veterans Affairs (Grants IK2-RX001479 and RX002705 to J.A.W.), National Institutes of Health/National Institute of Neurological Disorders and Stroke [Grants R01-NS-101108-01 (to J.A.W.) and T32-NS-043126 (to A.V.U.)] and the Codman Neurotrauma Award (P.F.K.).

P.F. Koch's present address: Department of Neurosurgery, Virginia Commonwealth University School of Medicine, Richmond, VA 23298; and Hunter Holmes McGuire Veterans Affairs Medical Center, Richmond, VA 23249.

Acknowledgments: We thank Brian N. Johnson and Kari L. Hoffman for comments on earlier versions of this manuscript.

Correspondence should be addressed to John A. Wolf at wolfjo@ pennmedicine.upenn.edu.

https://doi.org/10.1523/ENEURO.0495-19.2020

Copyright @ 2020 Koch et al.

This is an open-access article distributed under the terms of the Creative Commons Attribution 4.0 International license, which permits unrestricted use, distribution and reproduction in any medium provided that the original work is properly attributed. with spatial memory deficits, as well as early evidence that the exogenous restoration of theta may rescue memory performance (Fedor et al., 2010; Lee et al., 2013, 2015). However, others have found only a nonspecific, broadband reduction in hippocampal oscillatory power after injury (Paterno et al., 2016). It is unknown whether alterations in hippocampal oscillatory power after TBI reflect changes in specific synchronized synaptic inputs and lead to a change in hippocampal neuronal firing patterns, or whether power losses are nonspecific and potentially related to overall reductions in local neuron firing rates. We therefore used high-density silicon probes to record both the LFP and local neuron activity at multiple depths simultaneously across the anatomic laminar structure of CA1. This methodology allowed us to localize activity to specific layers (allowing identification of specific afferent projections into CA1) as well as to explore the relationship between synchronized synaptic input and local neuronal firing patterns after TBI. Given that the medial septum (MS) is implicated in the generation and maintenance of theta oscillations (Müller and Remy, 2018), we also explored whether communication between the hippocampus and the reciprocally connected medial septum was altered after TBI.

At 1 week following the most common rodent model of $\mathrm{TBI}$, we found frequency and anatomic layer-specific losses of oscillatory power in CA1 under anesthesia. Lowfrequency power losses were consistent with reduced or disorganized synaptic input into stratum radiatum (SR), but preserved theta oscillations, while high-frequency oscillatory power losses were restricted to the pyramidal cell layer. Single CA1 neurons maintained normal firing rates in the face of attenuated input after TBI, revealing that a subset of CA1 neurons can reach normal firing capacity. However, their ability to properly synchronize to dominant synaptic input and to local high-frequency oscillations was impaired. Single GABAergic medial septal neurons demonstrated increased firing rates after TBI and a reduced ability to synchronize to CA1 synaptic input, despite preserved synchronization with CA1 theta oscillations, suggesting impaired CA1 feedback. These findings support the hypothesis that oscillatory power losses after TBI reflect disruptions in anatomically specific synaptic inputs into the hippocampus, potentially affecting the organization of CA1 ensembles that underlie aspects of learning and memory dysfunction following TBI.

\section{Materials and Methods}

Details of resources used in this study are provided in Table 1.

\section{Animal care}

All animal procedures were performed in accordance with the regulations of the University of Pennsylvania animal care committee. Young, healthy, adult male Sprague Dawley rats (RGD catalog \#734476, Charles River Laboratories; RRID:RGD_734476) naive to any previous procedures or investigations were used in this study. Rats were chosen based on weight (range, 345-425 g, median weight, $383.5 \mathrm{~g}$ ). Animals were initially pair housed but 
Table 1: Key resources

\begin{tabular}{|c|c|c|}
\hline Reagent or resource & Source & Identifier \\
\hline \multicolumn{3}{|l|}{ Antibodies } \\
\hline $\begin{array}{l}\text { N-terminal amino acids } 66-81 \text { of the } \\
\text { APP, Clone } 22 \mathrm{C} 11\end{array}$ & Millipore & $\begin{array}{l}\text { Catalog \#MAB348, Millipore; } \\
\text { RRID: AB_94882 }\end{array}$ \\
\hline $\begin{array}{l}\text { Ab246, the caspase-derived fragment of } \\
\alpha \text {-spectrin }\end{array}$ & $\begin{array}{l}\text { Courtesy of Dr. Robert Siman, University } \\
\text { of Pennsylvania }\end{array}$ & Oo et al. (2002) \\
\hline \multicolumn{3}{|c|}{$\begin{array}{l}\text { Chemicals, peptides, and recombinant } \\
\text { proteins }\end{array}$} \\
\hline Ketamine & West-Ward & Catalog \#NDC0143-9509-01 \\
\hline Xylazine & Akorn & Catalog \#NDC59399-110-20 \\
\hline Acepromazine & Clipper Distributing Company & Catalog \#NDC57319-604-04 \\
\hline Normal horse serum & Vector Laboratories & Catalog \#S-2000-20 \\
\hline Fluoro-Jade C & Sigma-Aldrich & Catalog \#AG325 \\
\hline Bupivacaine & Medline Industries & Catalog \#0409-1610-50 \\
\hline Glycopyrrolate & Hikma Pharmaceuticals & Catalog \#NDC0143-9682-01 \\
\hline Screw, size $2-56 \times 3 / 8 "$ & Small Parts & Catalog \#MPX-0256-06F-M \\
\hline Luer Lock hub & Becton Dickinson & Catalog \#305165 \\
\hline $\begin{array}{l}\text { Dentsply Caulk Orthodontic Resin } \\
\text { (powder, liquid) }\end{array}$ & BencoDental & Catalog \#1007-910, catalog \#1007-894 \\
\hline Low-toxicity silicon adhesive, Kwik-Sil & World Precision Instruments & Catalog \#KWIK-SIL \\
\hline A silk suture, 4/0RB1 30' & Ethicon US & Catalog \#K871H \\
\hline Buprenex & Par Pharmaceuticals & Catalog \#NDC 42023-179-01 \\
\hline Tungsten electrode & $\mathrm{FHC}$ & Catalog \#UEWSEGSEBNNM \\
\hline 32-channel silicon probe & NeuroNexus Technologies & Catalog \#A1X32-Poly2-5mm-50s-177-H32 \\
\hline Thomas tetrode electrode & Thomas RECORDING & Catalog \#AN000020 \\
\hline Optimax buffer & BioGenex & Catalog \#HK583-5KE \\
\hline \multicolumn{3}{|l|}{ Experimental models: organisms/strains } \\
\hline Sprague Dawley rats & Charles River Laboratories & Catalog \#734476; RRID:RGD_734476 \\
\hline \multicolumn{3}{|l|}{ Software and algorithms } \\
\hline FP302 & $\begin{array}{l}\text { http://amscien.com/AmsFluid.htm } \\
\text { Instruments }\end{array}$ & \\
\hline MATLAB, version R2017a & MathWorks & MATLAB; RRID:SCR_001622 \\
\hline FMAToolbox & http://fmatoolbox.sourceforge.net/ & FMAToolbox; RRID:SCR_015533 \\
\hline Chronux & http://chronux.org & Chronux; RRĪ̄D:SCR_005547 \\
\hline CSDPlotter, version 0.1.1 & https://github.com/espenhgn/CSDplotter & Pettersen et al. (2006) \\
\hline KlustaKwik & http://klusta-team.github.io/klustakwik/ & KlustaKwik; RRID:SCR_014480 \\
\hline Circular statistics & $\begin{array}{l}\text { https://es.mathworks.com/matlabcentral/ } \\
\text { fileexchange/10676-circular-statistics- } \\
\text { toolbox-directional-statistics }\end{array}$ & Circular statistics; RRID:SCR_016651 \\
\hline GraphPad Prism & http://www.graphpad.com/ & GraphPad Prism; RRID:SCR_002798 \\
\hline KlustaViewa & $\begin{array}{l}\text { https://github.com/klusta-team/klustaviewa } \\
\text { https://github.com/kwikteam/phy }\end{array}$ & \\
\hline Noldus Information Technology & https://www.noldus.com & Noldus; RRID:SCR_004074 \\
\hline $\begin{array}{l}\text { EthoVision XT automated tracking } \\
\text { software }\end{array}$ & https://www.noldus.com/ethovision-xt & EthoVision XT; RRID:SCR_000441 \\
\hline
\end{tabular}

were subsequently housed singly after the injury or sham procedure. Rats were provided standard rat chow and water ad libitum. They were kept on a strict reversed $12 \mathrm{~h}$ light/dark cycle. After injury, or sham surgery, animals were examined daily for signs of pain or distress.

\section{Experimental design}

Twenty-one rats underwent initial training on the Morris water maze (MWM) in two sessions $24 \mathrm{~h}$ before being randomized to undergo fluid percussion injury (FPI) or sham surgery. Rats found to have a dural breach or a subdural hematoma at the time of surgery were excluded. On postsurgery day 1 , rats underwent memory testing on the Morris water maze. On postsurgery day 7, five of the injured and five of the sham-operated rats were randomized to undergo acute electrophysiological recording under anesthesia followed by sacrifice. An additional 10 rats (five injured, five sham) were sacrificed on postsurgery day 7 for histologic examination without undergoing electrophysiological recordings. Behavioral testing, electrophysiological recordings, histologic analysis, and initial data processing and spike sorting were performed blinded to the group status of each rat.

\section{Morris water maze}

The MWM is a widely used test of spatial learning and memory (Vorhees and Williams, 2006). We chose to implement a particular MWM protocol designed to specifically test hippocampal-dependent spatial memory, as distinct from broader learning/memory paradigms, and 
which has been demonstrated to be sensitive to the lateral fluid percussion injury (Smith et al., 1991). The apparatus consisted of an aluminum and Plexiglas circular pool $2 \mathrm{~m}$ in diameter and $50 \mathrm{~cm}$ tall with the inner surfaces painted black. The pool was filled with $18^{\circ} \mathrm{C}$ water to a depth of $25 \mathrm{~cm}$. A black $11.5 \times 11.5 \mathrm{~cm}$ platform that is $24 \mathrm{~cm}$ tall was placed in a fixed location within the pool. The black color of the platform and interior of the pool made the platform invisible from the surface. The pool was contained within a room with various objects placed within it, easily seen from within the pool. These objects remain fixed throughout training and testing to provide external visual cues for the rats. A video camera was placed above the pool and an automated video tracking system (EthoVision XT, Noldus; RRID:SCR_000441) recorded the position of the rat.

\section{Preinjury training}

Rats were selected that had no signs of ocular defect or any other health concerns. Animals were placed in the pool at a random location against the side of the tank and then allowed to freely swim, with the idea that they can escape the water by finding the invisible submerged platform. On the afternoon of the day before injury or sham injury, each animal was placed in the pool and given $2 \mathrm{~min}$ to find the platform. If an animal did not find the platform within the $2 \mathrm{~min}$, they were placed on the platform and allowed to remain for $30 \mathrm{~s}$ on the initial trial and $15 \mathrm{~s}$ on subsequent trials. The starting location for each trial was randomly chosen from four positions situated $90^{\circ}$ apart. A total of 10 trials were performed for each rat during the initial session, and the times taken to find the platform (latency) were recorded. Animals were then returned to their home cages to rest overnight. On the morning of injury or sham surgery, each rat underwent an additional 10 trials in an identical manner.

\section{Postinjury testing}

Approximately $42 \mathrm{~h}$ after undergoing fluid percussion injury or sham surgery, surviving rats were then tested for platform memory in the pool. After removing the submerged platform, each animal was released in the pool and the swimming activity of the rat was recorded for two trials of $1 \mathrm{~min}$ each. Using the automated tracking software EthoVision XT (RRID:SCR_000441) by Noldus (RRID:SCR_004074), a virtual grid over the surface of the pool was constructed, consisting of five zones of increasing distances from the former position of the platform. Time to first crossing of the platform position was calculated as the latency time. A memory score was also calculated, as detailed by Smith et al. (1991). Briefly, time spent in zones closer to the platform position were weighted higher than time spent in zones further away. These weighted times were then summed to give a memory score. Higher scores indicate better memory performance.

\section{Fluid percussion injury and sham surgery}

At $2 \mathrm{~h}$ after the completion of MWM training, animals were weighed and anesthetized with $5 \%$ isoflurane. After being placed in a stereotaxic head holder and maintained at $3 \%$ isoflurane, ophthalmic ointment was applied to the eyes for vision protection during surgery. Body temperature was maintained with the use of a heating pad throughout the surgery. Subcutaneous injections of bupivacaine (2 mg/kg; catalog \#0409-1610-50, Medline Industries) and glycopyrrolate $(0.01 \mathrm{mg} / \mathrm{kg}$; catalog \#NDC 0143-9682-01, Hikma Pharmaceuticals) were administered followed by a midline incision and skull exposure. Next, a 5-mm-diameter craniectomy was performed over the left parietal cortex midway between bregma and lambda, with the center $\sim 2.5 \mathrm{~mm}$ lateral from midline, centering the defect between midline and the temporal ridge. Rats were maintained at $2-2.5 \%$ isoflurane. A screw (size, 2-56 × 3/8 inch; catalog \#MPX-0256-06F-M, Small Parts) was inserted anterior to bregma on the right hemisphere for additional structural support. Both FPI-injured and sham animals received a craniotomy and drill hole. Next, a Luer Lock hub (from a 21 gauge needle; catalog \#305165, Becton Dickinson) was secured to the craniotomy site with super glue (All Purpose Krazy Glue No Run Gel, Elmer's Products) followed by the application of dental cement [Dentsply Caulk Orthodontic Resin; catalog \#1007-910 (powder) and catalog \#1007-894 (liquid), BencoDental] to ensure the formation of a secure head cap before injury. The FPI device (AmScien Instruments) consisted of a Plexiglas, cylindrical, saline-filled reservoir bounded at one end by a Plexiglas plunger mounted on O-rings. The opposite end of the cylinder was capped with a male luer stub, which connected to the Luer Lock cemented on the craniotomy. After removal of anesthesia, animals were monitored for increased respiration, at which point they were attached to the device. For the injury group, once secured, a pendulum was dropped from $20^{\circ}$, striking the plunger and causing a rapid and highpressure injection of saline into the closed cranial cavity, compressing the brain. The injury level was measured in atmospheres (atm; $\mathrm{FPI}=2.3 \pm 0.1 \mathrm{~atm}$, mean $\pm \mathrm{SD}$ ) by a pressure transducer, and both the injury trace and atmospheres were recorded (catalog \#FP302, AmScien Instruments). For the sham surgery group, the pendulum was not dropped. Animals were then monitored for apnea and seizure postinjury and once stabilized were placed on $1 \%$ isoflurane. Before suturing, a low-toxicity silicon adhesive (catalog \#KWIK-SIL, World Precision Instruments) was applied to the craniotomy site to mitigate scarring, after which the incision was closed with a silk suture (4/0 RB1 30'; catalog \#K871H, Ethicon US). Animals were recovered under a heat lamp and administered a subcutaneous injection of Buprenex $(0.05 \mathrm{mg} / \mathrm{kg}$; catalog \#NDC 42 023-179-01, Par Pharmaceuticals) after demonstrating sufficient recovery of the righting reflex.

\section{Acute recording procedure}

On postinjury or post-sham surgery day 7 animals were anesthetized in a Plexiglas chamber with $5 \%$ isoflurane. They were then placed in a stereotaxic frame using standard ear bars (Stoelting), and the head was leveled under $3 \%$ isoflurane. Ophthalmic ointment was applied to the eyes for protection of the cornea during surgery. Body temperature was maintained with the use of a heating pad 
throughout the surgery. Periodic tail pinches were performed throughout the recording session to ensure an adequate depth of anesthesia. Subcutaneous injections of bupivacaine (2 mg/kg; catalog \#0409-1610-50, Medline Industries) and glycopyrrolate $(0.01 \mathrm{mg} / \mathrm{kg}$; catalog \#NDC 0143-9682-01, Hikma Pharmaceuticals) were administered. The skull was then exposed and leveled with respect to bregma and lambda suture landmarks per the stereotaxic methods of Paxinos and Watson (2007). A skull screw was placed over the right cerebellum to serve as an animal ground. A tungsten electrode $(\varnothing, 125 \mu \mathrm{m}$; length, $60 \mathrm{~mm}$; impedance, $0.5 \mathrm{M} \Omega$; catalog \#UEWSEGSEBNNM, FHC) with the tip clipped and stripped was inserted into the right lateral ventricle [anteroposterior (AP), 2.0; ML, 1.6; DV, 3.45] to serve as an intracranial reference signal. We then expanded the previously created craniectomy over the left frontoparietal cortex and created a second craniectomy crossing midline, eccentric to the left and just anterior to bregma. A 32-channel silicon probe (catalog \#A1X32-Poly2$5 \mathrm{~mm}-50 \mathrm{~s}-177-\mathrm{H} 32$, NeuroNexus) was then slowly inserted into the left dorsal hippocampus (AP, -4.2; ML, 2.7). Signals from all 32 channels were amplified and visualized in real time during insertion and monitored both for changes in the LFP and spiking activity. Once spiking activity representing the pyramidal cell layer of CA1 was identified on the distalmost channels, this activity was followed proximally along the channels of the probe as it was inserted deeper until it was detected in the proximal third of the probe (final depth range, $2.73-4.19 \mathrm{~mm}$; median, $3.19 \mathrm{~mm}$ ). The isoflurane was then lightened to the minimum dose required for no response from a tail pinch (range, 1.5-2). After a 30 min period, during which isoflurane levels and signals were allowed to reach a steady state, a 15 min recording was performed. A tetrode (catalog \#AN000020, Thomas RECORDING) was then inserted into the left medial septum (AP, 0.6; ML, 0.5; lateral to medial angle of $5^{\circ}$ ). Spiking activity was monitored, and 5 min recordings were made at each depth below 5.2 $\mathrm{mm}$ at which spiking activity could be isolated (minimum of $100 \mu \mathrm{m}$ separation).

At the end of the electrophysiological recording procedure, the level of isoflurane anesthesia was raised to $5 \%$. After checking for pain responses, the animal was sacrificed by injecting ketamine $(75 \mathrm{mg} / \mathrm{kg}$; catalog \#NDC 0143-9509-01, West-Ward), xylazine (15 mg/kg; catalog \#NDC 59399-110-20, Akorn), and acepromazine (2 mg/ kg; catalog \#NDC 57319-604-04, Clipper). The animal underwent transcardial perfusion with $0.9 \%$ heparinized saline followed by $10 \%$ neutral buffered formalin (NBF), and the brain was extracted in a manner identical to that in the nonelectrophysiology cohorts (see Histologic examinations section).

\section{Data acquisition}

All electrophysiological recordings were made under isoflurane anesthesia. Neural signals were amplified and acquired continuously at $32 \mathrm{kHz}$ on a 64-channel Digital Lynx 4SX acquisition system (Neuralynx). Raw signals were filtered from $0.1 \mathrm{~Hz}$ to $9 \mathrm{kHz}$ on the acquisition board, digitized, and saved. The filtered signal was also displayed for real-time inspection of the LFP during recordings. The raw signals were bandpass filtered from 600 to $6000 \mathrm{~Hz}$ for real-time spike inspection. Spike thresholds were manually adjusted during recordings for real-time display purposes based on observed signal-tonoise ratios during the recording session.

\section{Histologic examinations}

A separate subset of animals underwent FPI $(n=4)$ or sham surgery $(n=5)$ only (no electrophysiology) to permit neuropathological examination of the model without the confounding pathology caused by electrode implantation. All animals survived for $7 \mathrm{~d}$ and were otherwise handled under protocols identical to those animals that underwent electrophysiological examinations. At the study endpoint, a mixture of ketamine $(75 \mathrm{mg} / \mathrm{kg}$; catalog \#NDC 01439509-01, West-Ward), xylazine (15 mg/kg; catalog \#NDC 59 399-110-20, Akorn), and acepromazine (2 mg/kg; catalog \#NDC 57319-604-04, Clipper) was administered. Following adequate anesthesia, animals were transcardially perfused with an initial flush of chilled and heparinized $1 \times$ PBS followed immediately with $200 \mathrm{ml}$ of chilled $10 \%$ NBF. Brains were extracted and postfixed for $24 \mathrm{~h}$ at $4^{\circ} \mathrm{C}$ before being blocked in the coronal plane at $2 \mathrm{~mm}$ intervals and processed to paraffin using standard techniques. Eight-micrometer-thick sections were obtained every $2 \mathrm{~mm}$ throughout the rostrocaudal extent of the brain using a rotary microtome. To permit characterization and verify reproducibility of the injury model, tissue sections were first stained with hematoxylin and eosin (H\&E) as described previously (Johnson et al., 2013a).

\section{Immunohistochemistry}

To examine for the presence and distribution of axonal pathology and cell death, standard immunohistochemistry (IHC) techniques were performed as previously described (Johnson et al., 2013a, 2016, 2018). Briefly, following deparaffinization and rehydration of tissue sections, endogenous peroxidase activity tissue was quenched using $3 \%$ aqueous hydrogen peroxide (15 min). Antigen retrieval was performed using a microwave pressure cooker with sections immersed in Tris EDTA buffer, pH 8.0. Subsequent blocking was performed for $30 \mathrm{~min}$ in $1 \%$ normal horse serum (Vector Laboratories) in Optimax buffer (BioGenex). Incubation with the primary antibodies was performed at $4^{\circ} \mathrm{C}$ overnight. Specifically, to identify axonal pathology, sections were labeled with an antibody reactive for the $\mathrm{N}$-terminal amino acids 66-81 of the amyloid precursor protein (APP; 1:130,000; Clone 22C11, Millipore; Gentleman et al., 1993; Johnson et al., 2016) and Ab246 specific for the caspase-derived fragment of $\alpha$-spectrin, indicative of apoptosis (1:17,000; Bakshi et al., 2005; Chen et al., 2005; Oo et al., 2002; courtesy of Robert Siman, University of Pennsylvania). After rinsing, sections were incubated with the appropriate biotinylated secondary antibody for $30 \mathrm{~min}$ followed by avidinbiotin complex and visualization achieved using DAB (all reagents are from Vector Laboratories). Sections were rinsed and dehydrated in graded alcohols and cleared in xylenes before being coverslipped. Positive 
control tissue for APP IHC included sections of contused rat tissue with previously established axonal pathology. Omission of the primary antibodies was performed on positive control tissue to control for nonspecific binding.

\section{Fluoro-Jade C staining}

As an additional marker of degenerating neurons, Fluoro-Jade C staining was performed (Schmued et al., 2005). Specifically, following dewaxing and rehydration to water as above, tissue was the immersed in a $0.06 \%$ potassium permanganate solution for $20 \mathrm{~min}$ at room temperature. After being rinsed in gently flowing distilled $\mathrm{H}_{2} \mathrm{O}$, tissue was then incubated in $0.00012 \%$ Fluoro-Jade C solution (in $0.1 \%$ acetic acid) at room temperature for $30 \mathrm{~min}$. After rinsing, sections were dried at $37^{\circ} \mathrm{C}$ for 90 min before being immersed in xylenes and coverslipped as above.

\section{Analysis of histologic findings}

Axonal pathology was examined within a tissue section at the level of the hippocampus (AP, -2.76) and included specific assessment of the fimbria fornix, angular bundle, stratum radiatum, stratum lacunosum-moleculare (SL-M), and stratum oriens (SO) of the hippocampus. The medial septum was also examined at $A P+0.60$. In addition, cell death was assessed by examination for Fluoro-Jade C and Ab246-positive cells in the medial septum, CA1 and CA3 subfields, and stratum oriens of the hippocampus. The presence or absence of pathology was recorded. A high level of inter-rater reliability in the identification of pathology by two independent observers was achieved (Cohen's kappa, 0.98).

\section{Statistics}

All statistical analyses were performed in Prism 7 or 8 (GraphPad Prism; RRID:SCR_002798) or using the MATLAB Circular Statistics Toolbox (circular statistics; RRID:SCR_016651). Samples were reported as either numbers of rats or numbers of cells. When samples were numbers of rats, we reported the median and range. When samples were numbers of cells, we reported means and SDs. The Welch's $t$ test, or Mann-Whitney test was used for mean or median group comparisons, respectively. All plots with error bars depict the mean \pm SD or median with interquartile ranges, unless otherwise indicated. In the case of LFP power comparisons between groups, the repeated-measures ANOVA was used, and plots with error bars of these data are reported as the mean \pm SEM. In the cases of comparing modulation index (MI) values between groups (phase amplitude coupling and single-unit entrainment to high-frequency oscillations), comparisons were made using the Student's $t$ test and plots with error bars of these data are reported as the mean \pm SEM.

\section{Electrophysiology data analysis}

Raw signals recorded with the 32-channel silicon probe were downsampled to $2 \mathrm{kHz}$ for LFP analysis. Signals were imported into MATLAB software, version R2017a (MATLAB;
RRID:SCR_001622) and processed using a combination of custom and modified routines from the freely available MATLAB packages FMAToolbox (FMAToolbox; RRID:SCR 015533) and Chronux (RRID:SCR_005547; Hazan et al., 2006; Mitra and Bokil, 2008).

\section{Channel alignment}

For comparative analysis of neurophysiological signals between the injured and sham groups of animals, we first bandpass filtered $(0.6-6 \mathrm{kHz})$ the LFP for each channel of the 32-channel $\mathrm{HC}$ probe, giving an estimate of the multiunit spiking activity. We then selected the channel with the maximum average power for each animal and designated that channel to be the pyramidal cell layer. HC probes from all animals were then aligned to this channel. This procedure resulted in 25 aligned channels of neurophysiological data common to all animals.

\section{Current source density}

Current source density (CSD) of the LFP across all HC channels was calculated with the spline inverse CSD method of Pettersen et al. (2006), using the freely available MATLAB package CSDplotter version 0.1.1 (https:// github.com/espenhgn/CSDplotter) visualized by convention as a heat map of current sinks (blue) and sources (orange; Mitzdorf, 1985).

\section{LFP power}

To investigate any statistical differences in power between the two groups and among each of the 25 channels, we windowed each $15 \mathrm{~min}$ recording in $1 \mathrm{~s}$ epochs and calculated the power spectral density in the $1-300 \mathrm{~Hz}$ frequency band (Welch's method: frequency resolution, $0.5 \mathrm{~Hz}$; overlap, 60\%) for each epoch. As a result, we obtained a 25 (channels) $\times$ 600 (frequency bins) $\times 2250$ (epochs) matrix of power values for each subject. We then calculated the channel-frequency map power difference distribution (PDD) relative to the sham group [(injured/sham - 1)\%], averaging the aforementioned matrix along the epochs and groups. To evaluate where these differences were statistically significant, we calculated a significance mask through a repeated-measures ANOVA between the two groups using the epochs as independent variables, obtaining a channel-frequency map of $p$ values: $p$ values $<0.05$ were considered significant and converted to a value of 1 , otherwise values were represented with a 0 . Finally, we multiplied the significance mask by the PDD to identify where the two groups displayed statistically significant differences.

\section{Phase amplitude coupling}

To investigate the interaction between oscillations in different frequency bands after injury, we used phaseamplitude coupling (PAC) as a form of cross-frequency coupling analysis in the HC. We investigated how the phase of lower-frequency oscillations drive the power of coupled higher-frequency oscillations by measuring the degree of synchronization of the amplitude envelope of faster rhythms with the phase of slower rhythms. To quantify the PAC between strata radiatum and pyramidale LFP, we used the Kullback-Leibler (KL)-based MI based on the joint entropy (Tort et al., 2010). 
Briefly, Ml was calculated as follows: components of the stratum pyramidale (SP) LFP for each frequency $[1-300 \mathrm{~Hz}$, denoted as $\left.\operatorname{Pyr}\left(t, f_{\mathrm{pyr}}\right), f_{\mathrm{pyr}}=1,2,3 \ldots 299,300 \mathrm{~Hz}\right]$ were extracted by wavelet transform (Morlet; 10 cycles). The phases of the bandpass filtered $(1-20 \mathrm{~Hz})$ stratum radiatum LFP (denoted as $\Phi_{\operatorname{Rad}\left(t, f_{\text {rad }}\right)}, f_{\text {rad }}=1,2,3 \ldots .20 \mathrm{~Hz}$ ) were obtained from the standard Hilbert transform. The Hilbert transform was also applied to extract time series of the amplitude envelopes (denoted as $\left.A_{\text {Pyr }\left(t, f_{\mathrm{pyr}}\right)}\right)$ of $\operatorname{Pyr}\left(t, f_{\mathrm{pyr}}\right)$. The composite time-frequency series $\left(\Phi_{\operatorname{Rad}\left(t, f_{\mathrm{rad}}\right)}, A_{\mathrm{Pyr}\left(t, f_{\mathrm{pyr}}\right)}\right)$ was then constructed, which gave the amplitude of the pyramidal layer LFP for each frequency bin oscillation $(1-300 \mathrm{~Hz})$ at each phase of the radiatum layer LFP for each frequency bin $(1-20 \mathrm{~Hz})$. Next, the phases $\Phi_{\operatorname{Rad}\left(t, f_{\mathrm{rad}}\right)}$ were binned and the mean value of $A_{\text {Pyr }\left(t, f_{\text {prr }}\right)}$ over each binned phase was calculated. We denoted $\left[A_{\text {Pyr }\left(t, f_{\mathrm{pyr}}\right)}\right] \Phi_{\operatorname{Rad}\left(t, f_{\text {rad }}\right)}(j)$ for the mean $A_{\text {Pyr }\left(t, f_{\mathrm{pyr}}\right)}$ value at the phase bin $j$. Afterward, the normalized mean amplitude of $\left[A_{\text {Pyr }\left(t, f_{\text {pyr }}\right)}\right] \Phi_{\operatorname{Rad}\left(t, f_{\text {rad }}\right)}(j)$ was divided for each bin value by the sum over all bins. The phase-amplitude distribution for each combination $\left(f_{\mathrm{pyr}}, f_{\mathrm{rad}}\right)\left(P\left(j, f_{\mathrm{pyr}}, f_{\mathrm{rad}}\right)\right)$ was obtained by the following equation, where $N$ was the number of phase bins:

$$
\begin{gathered}
P\left(j, f_{\text {pyr }}, f_{\text {rad }}\right)=\frac{\left[A_{\text {Pyr }\left(t, f_{\text {pyr }}\right)}\right] \Phi_{\text {Rad }\left(t, f_{\text {rad }}\right)}(j)}{\sum_{j=1}^{N}\left[A_{\text {Pyr }\left(t, f_{\text {pyr }}\right)}\right] \Phi_{\text {Rad }\left(t, f_{\text {rad }}\right)}(j)} \\
f_{\text {pyr }}=1,2,3 \ldots .299,300 \mathrm{~Hz}, f_{\text {rad }}=1,2,3 \ldots 20 \mathrm{~Hz},
\end{gathered}
$$

The joint entropy $(H)$ of $\operatorname{Rad}\left(t, f_{\text {rad }}\right)-\operatorname{Pyr}\left(t, f_{\text {pyr }}\right)$ coupling was calculated as follows:

$$
H\left(f_{\mathrm{pyr}}, f_{\mathrm{rad}}\right)=-\sum_{j=1}^{N} P\left(j, f_{\mathrm{pyr}}, f_{\mathrm{rad}}\right) \log P\left(j, f_{\mathrm{pyr}}, f_{\mathrm{rad}}\right) .
$$

When the joint distribution of $\Phi_{\operatorname{Rad}\left(t, f_{\text {rad }}\right)}-A_{\text {Pyr }\left(t, f_{\text {pyr }}\right)}$ was uniform, the maximum value of joint entropy $H_{0}$ was obtained, as follows:

$$
H_{0}=\log N^{2} .
$$

We then calculated the $\mathrm{KL}$ distance by subtracting the value $H_{0}$ from $H$, and measured how much the $\Phi_{\operatorname{Rad}\left(t, f_{\mathrm{rad}}\right)}-$ $A_{\text {Pyr }\left(t, t_{\text {pyr }}\right)}$ joint distribution deviated from the uniform joint distribution. Finally, MI was obtained by dividing the $\mathrm{KL}$ distance by the constant factor $H_{0}$, which made the measure assume values between 0 and 1 , as follows:

$$
\operatorname{MI}\left(f_{\text {pyr }}, f_{\text {rad }}\right)=\frac{H\left(f_{\text {pyr }}, f_{\text {rad }}\right)-H_{0}}{H_{0}} .
$$

Thus, a larger MI value denoted a stronger coupling between the stratum radiatum and stratum pyramidale layers of the $\mathrm{HC}$.

To investigate any statistical differences in coupling between the two groups, we calculated the $t$ test of $\operatorname{Ml}\left(f_{\text {pyr }}, f_{\text {rad }}\right)$ for each combination of $f_{\text {pyr }}$ and $f_{\text {rad }}$.

\section{Spike sorting}

Automated offline spike detection and sorting were performed on the wideband signals using the Klusta package
(KlustaKwik; RRID:SCR_014480), developed for higherdensity electrodes, and manually refined using the KlustaViewa (https://github.com/klusta-team/klustaviewa), or phy (https://github.com/kwikteam/phy) software package. The Klusta routines are designed to take advantage of the spatial arrangement of and spike timing from all probe channels simultaneously (32 channels for $\mathrm{HC}$ probe, 4 channels for MS tetrode) in constructing putative clusters (Rossant et al., 2016). Putative single units were required to have at least 100 spikes and a refractory period violation rate of $<2 \%$ (interspike interval of $<3 \mathrm{~ms}$ ). For putative units isolated on the 32-channel HC probe, we further required a quality index of $>0.95$, a measure of cluster uniqueness relative to all other potential clusters in the dataset computed by the KlustaViewa software (Rossant et al., 2016). For putative units isolated on the MS tetrode, we additionally required the isolation distance (Harris et al., 2001) of each putative single unit to be $\geq 20$ (Harris et al., 2003). The resulting curated putative single-unit clusters were then imported into MATLAB software, version R2017a, for visualization and further analysis using custom and built-in routines (MATLAB; RRID:SCR_001622). Wideband signals were bandpass filtered $(0.6-6 \mathrm{kHz})$ for average waveform visualization only.

\section{Subtype classification of putative single units in CA1}

We created a three-step process for classifying isolated $\mathrm{HC}$ putative single units on the 32-channel probe as either pyramidal cells or interneurons. In the first step, putative units were assigned to an anatomic layer (stratum oriens, stratum pyramidale, or stratum radiatum). We defined a five-channel span $(100 \mu \mathrm{m})$ centered on the channel of maximum multiunit activity for each rat and defined this as stratum pyramidale. For each putative unit, if the channel of maximum amplitude fell within this band, it was assigned to stratum pyramidale. If the maximum amplitude was above this band, it was assigned to stratum oriens, and if below, it was assigned to stratum radiatum. If the maximum amplitude of a putative unit fell on a channel adjacent to the stratum pyramidale band, it was classified temporarily as a border unit. All nonborder putative units outside of stratum pyramidale were then classified, by definition, as interneurons, regardless of firing rate. In the second step, we modified the method of Csicsvari et al. (1999) of putative unit classification into pyramidal cells, or interneurons and applied it to all units assigned to stratum pyramidale or classified as border units. This approach computes the following three features of each putative unit: overall firing rate, a measure of averaged spike waveform width, and a measure of "burstiness" in the spike train of the unit. Spike width was calculated by extracting the waveform for each spike from the unfiltered wideband signal, which was then averaged and upsampled by a factor of 5 . The peak amplitude of the averaged waveform was then detected, and its height was calculated by subtracting a baseline amplitude $1 \mathrm{~ms}$ before the peak. The spike width was then calculated as the time interval between the points along the averaged waveform that fell at $25 \%$ of the spike height, centered on the peak. Burstiness was defined as the first moment of the autocorrelogram, with smaller values indicating higher burstiness. The distributions of these three features 
across all putative stratum pyramidale units were plotted and inspected for natural clusters. Each feature revealed two clusters, and we thus made a simple division into two clusters for each feature using threshold values (see Fig. $4 B$ ). Putative pyramidal cells were defined as those units with a spike width above the determined threshold, a first moment of the autocorrelogram below the determined threshold (equating to high burstiness), and a firing rate below the determined threshold (division was at $3.5 \mathrm{~Hz}$ ). Similarly, putative interneurons were defined as those units with a spike width below threshold, a first moment above threshold (equating to low burstiness), and a firing rate $>3.5 \mathrm{~Hz}$. In a final third step, those putative units classified as both interneurons and border cells were reassigned to stratum pyramidale if there were also isolated putative pyramidal cells with maximum amplitudes on the same channel, otherwise they were assigned either to stratum oriens or stratum radiatum, depending on which border they were located.

\section{Subtype classification of putative single units in medial septum}

To classify isolated single units in the MS as either putative GABAergic/glutamatergic (GABA/Glu) or putative cholinergic, we modified a method first described using intracellular and juxtacellular recordings in slice preparations (Matthews and Lee, 1991) and subsequently extended to in vivo recordings (King et al., 1998). This method relies solely on waveform shape by capturing an observed "inflection" of the repolarization phase of the action potential found to be characteristic of cholinergic neurons, but not GABAergic/glutamatergic neurons. Spectral power analysis of the waveforms from 1 to $16 \mathrm{kHz}$ reveals increased power in higher-frequency bins in the "inflected" waveform group. We found that the distribution of the ratio of the fourth to second frequency bin revealed two natural clusters in both the sham and injured groups. The lower ratio, or "noninflected," waveform cluster was then classified as putative GABAergic/glutamatergic cells. The higher ratio, or inflected, waveform cluster was then further refined by firing rate, as we expect cholinergic units to have firing rates generally $<4 \mathrm{~Hz}$. The firing rate distribution of the inflected subgroup revealed a low-firing cluster that was then classified as the putative cholinergic subgroup.

\section{Entrainment of single units to local field potentials}

For entrainment of putative $\mathrm{HC}$ or MS single units to the low-frequency oscillations, we bandpass filtered [8-20 or $2-5 \mathrm{~Hz}$ ("anesthesia theta")] the LFP of the channel $425 \mu \mathrm{m}$ below the center of stratum pyramidale, which corresponded to the channel of maximal power difference between groups within the $8-20 \mathrm{~Hz}$ range, and extracted the phase angles for each spike within each putative single unit using the Hilbert transform. Directional statistics were performed using MATLAB Circular Statistics Toolbox (CircStat; RRID:SCR_016651). For each putative unit, the mean resultant vector was calculated, giving the mean phase angle of entrainment for that unit, as well as the resultant vector length, a measure of spread of the individual phase angles about the mean. Putative units were then considered significantly entrained if the resultant vector length was greater than the threshold of nonuniform distribution calculated by the Rayleigh test (Berens, 2009). The mean angles of entrainment between significantly entrained sham and injured putative units as well as their distributions were compared using the twosample Watson-Williams test and the two-sample Kuiper test, respectively.

For entrainment of putative $\mathrm{HC}$ single units to fast oscillations in stratum pyramidale we first extracted components of the stratum pyramidale LFP by wavelet transform (Morlet, 10 cycles) for each frequency $(1-400 \mathrm{~Hz}$, denoted as $\operatorname{Pyr}(t, f), f=1,2,3, \ldots 399,400 \mathrm{~Hz})$. The phases for each frequency bin (denoted as $\Phi_{\operatorname{Pyr}(t . f)}, f=1,2,3, \ldots 399,400$ ) were obtained from the standard Hilbert transform. Next, the phases $\Phi_{\mathrm{Pyr}(t, f)}$ were binned (18 bins, $\left.20^{\circ}\right)$, and the number of units per bin [\#units())] was calculated. We denoted the normalized unit distribution over the phase bin $j$ as follows:

$$
P(j, f)=\frac{\# \text { units }(j)}{\sum_{j=1}^{N} \# \text { \#nits }(j)} .
$$

The joint entropy $H$ was calculated as follows:

$$
H(f)=-\sum_{j=1}^{N} P(j, f) \log P(j, f) .
$$

We then calculated the MI for putative single unit-stratum pyramidale LFP entrainment, as per methods detailed above (see Phase-amplitude coupling section). A larger $\mathrm{Ml}$ value denoted a stronger entrainment. As above, we used the Student's $t$ test to determine frequencies of significant differences in MI.

\section{Spike-triggered averages}

To investigate the influence of $\mathrm{HC}$ oscillatory activity on MS neuron firing patterns, we computed MS spike-triggered averages of the HC LFP recorded simultaneously with spiking activity in the MS. For each putative MS neuron, we averaged $1 \mathrm{~s}$ windows of the unfiltered HC LFP centered on each putative MS cell spike. Spike-triggered averages of putative units meeting criteria for "CA1 modulated" (see below) were then averaged within the sham and injured groups. To quantify differences in the averaged spike-triggered averages between the sham and injured groups, we calculated the absolute difference between the maximum positive and maximum negative voltage deflections in the averaged tracings for each unit, making between-group comparisons using Welch's $t$ test.

\section{Peristimulus time histograms}

For each putative CA1-modulated MS unit the concurrently recorded HC LFP within stratum radiatum was bandpass filtered from 8 to $20 \mathrm{~Hz}$ or from 2 to $5 \mathrm{~Hz}$ (anesthesia theta). The troughs of the resulting oscillation were detected, and the times of these events were used as a trigger for the creation of the peristimulus time histogram (PSTH; $100 \mathrm{~ms}$ window; bin size, $2 \mathrm{~ms}$ ) for each putative CA1-modulated MS unit. Because individual MS units 
had different overall firing rates for each bin, the number of spikes was divided by the total number of spikes for that putative unit to derive a firing probability that could be compared across units.

\section{Data availability}

Custom MATLAB scripts written for electrophysiology data analysis are described above. Custom scripts and data presented in this article are available on request.

\section{Results}

\section{Injury physiology and behavioral deficits confirm moderate to severe TBI}

The median delivered pressure wave during lateral FPI in the injured group $(N=11)$ was $2.30 \mathrm{~atm}$ (range, 2.01$2.46 \mathrm{~atm}$ ), and the median apnea time after injury was $27 \mathrm{~s}$ (range, 0-105 s), consistent with a moderate to severe level of injury (Smith et al., 1991). One animal had a $5 \mathrm{~s}$ seizure in the immediate postinjury period. One animal experienced a large subdural hematoma after FPI and was excluded. No dural breaches occurred.

Both the injured group $(N=10)$ and sham group $(N=10)$ underwent preinjury training and postinjury testing (postinjury day 1) on the Morris water maze. The median calculated memory score for recall of the hidden platform during postinjury testing was significantly lower in the injured group compared with the sham group [injured group: 21.21 (range, 0-86.35); sham group: 65.84 (range, 4.67-113.9); Mann-Whitney test, $p=0.012$ ], while there was no difference in swimming speed (Mann-Whitney test, $p=0.74$ ). These hippocampus-relevant performance deficits are also consistent with a moderate to severe injury (Smith et al., 1991).

\section{Hippocampal power losses after FPI are anatomic layer and frequency band specific}

Five animals each from the sham and injured groups underwent acute recordings from the hippocampus under isoflurane anesthesia [median isoflurane levels: sham group, 1.63 (range, 1.5-2); injured group, 1.5 (range, 1.51.75); Mann-Whitney test, $p=0.76$ ] $7 \mathrm{~d}$ after FPI or sham injury. A 32-channel silicon probe (Poly-2 design) capable of recording LFP and single-unit activity was inserted into the dorsal CA1 region of the ipsilateral hippocampus, spanning multiple anatomic layers, including stratum oriens (SO), stratum pyramidale (SP), stratum radiatum (SR), and a portion of stratum lacunosum-moleculare (SL-M) (Fig. $1 A, D, J)$.

We first examined whether there were LFP power differences between the sham and injured groups that were frequency specific and anatomic layer specific. To quantify this, we calculated the power spectral density at each anatomically distributed recording channel from 1 to $300 \mathrm{~Hz}$ for each animal. Channels were then spatially aligned to the channel of peak spiking activity within SP (see Materials and Methods), giving a total of 25 channels of overlap extending from SO to the bottom of SR. We compared the power density between the sham and injured groups at each channel and frequency, generating a map of significant differences (repeated-measures ANOVA, $p<0.05$ ). Although there were no regions of increased overall oscillatory power after injury, the power was significantly reduced within SR, with peak reduction of $46.7 \%$ at a frequency of $13.5 \mathrm{~Hz}$ (range, $10.5-43 \mathrm{~Hz}$ ) at its deep extent, and within SO with peak reduction of $45 \%$ at a frequency of $26 \mathrm{~Hz}$ (range, $10-39 \mathrm{~Hz}$; Fig. $1 \mathrm{G}, /$ ).

To better characterize this $\sim 10-15 \mathrm{~Hz}$ SR/SO oscillation, we visually inspected the unfiltered LFP in sham and injured rats. The sham LFP revealed a prominent oscillation in the $\sim 10-15 \mathrm{~Hz}$ range [median frequency of peak power: $12 \mathrm{~Hz}$ (range, $10-15 \mathrm{~Hz}$ )] detectable at all recording sites spanning the $\mathrm{CA} 1$ anatomic layers, but with greatest amplitude in SR (Fig. 1A). This oscillation was present with a $180^{\circ}$ phase reversal within SO. To further refine the anatomic specificity of this oscillation, the CSD was calculated for representative segments of the LFP exhibiting this oscillation, revealing a prominent sink centered in SR and an associated source in SP (Fig. 1B). Inspection of the LFP and associated CSD in the injured animals revealed a similar oscillation but with an apparent lower amplitude (Fig. 1D,E). The median frequency of peak power in the injured group was $9.5 \mathrm{~Hz}$ (range, 9$12 \mathrm{~Hz}$; Mann-Whitney test, $p=0.09$ vs sham). Together, these data suggest that there is a prominent coordinated synaptic input into SR in both the sham and injured groups, but that the strength of this input is significantly reduced after injury, either because of reduced presynaptic input, reduced coordination of that input, or both.

Although present equally in sham and injured animals, a second prominent power peak was observed in SR within the $\sim 2-5 \mathrm{~Hz}$ range [median frequency, $2 \mathrm{~Hz}$ (sham and injured groups); Mann-Whitney test, $p=0.64$; Fig. 1/]. While hippocampal theta oscillations in the awake, exploring rat are in the $4-10 \mathrm{~Hz}$ range, it has been reported that oscillations with a similar laminar pattern within rodent CA1 can be observed under isoflurane anesthesia in the $2-5 \mathrm{~Hz}$ range (Lustig et al., 2016), here termed anesthesia theta. To further explore whether these $\sim 2-5 \mathrm{~Hz}$ oscillations were likely to represent anesthesia theta oscillations (analogous to awake theta oscillations), we filtered the wideband LFP from 2 to $5 \mathrm{~Hz}$ and located epochs of increased power within this band for inspection of the laminar profile. A representative $1 \mathrm{~s}$ epoch of LFP and CSD illustrating prominent anesthesia theta from the sham and injured groups are depicted in Figure 1, $C$ and $F$. These oscillations demonstrate the largest amplitude coupled with the maximal CSD sink within SL-M, suggesting that significant coordinated synaptic input into SL-M is present (Fig. 1C,F). There is also a progressive phase shift of this oscillation from SO to SL-M. These features are characteristic of $4-10 \mathrm{~Hz}$ theta oscillations during the awake state (Buzsáki, 2002) and also observed in the 2$5 \mathrm{~Hz}$ range under isoflurane anesthesia (Lustig et al., 2016). As peak power of theta oscillations occurs in SL-M (Buzsáki, 2002), it is possible we failed to capture a difference in theta power in the aligned channel data, as the deepest channel was still located within SR. We therefore compared the spectral power between subgroups of the sham $(n=3)$ and injured $(n=4)$ cohorts that had recording 
A
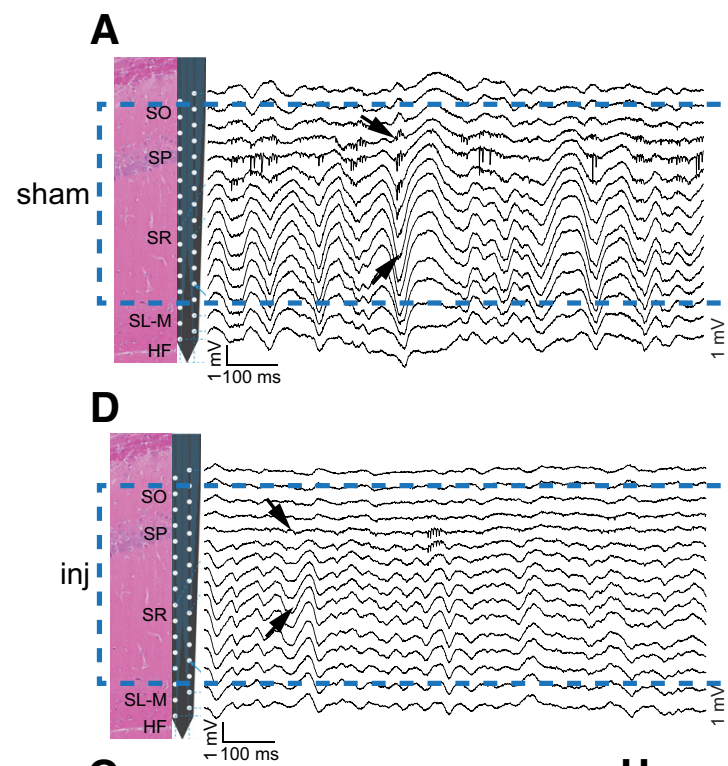

G

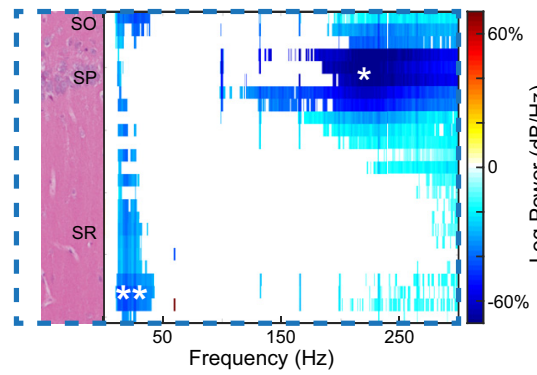

B

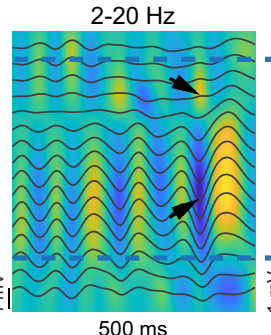

E

$2-20 \mathrm{~Hz}$

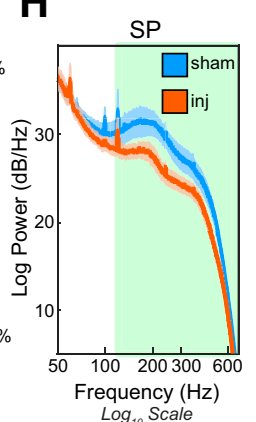

C

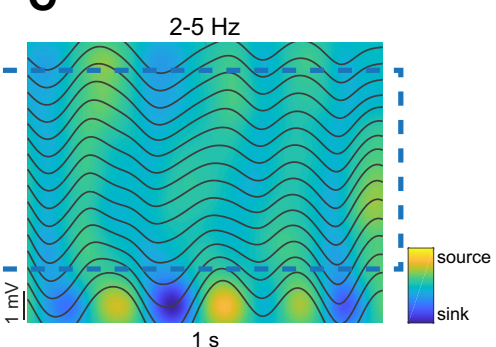

$\mathbf{F}$

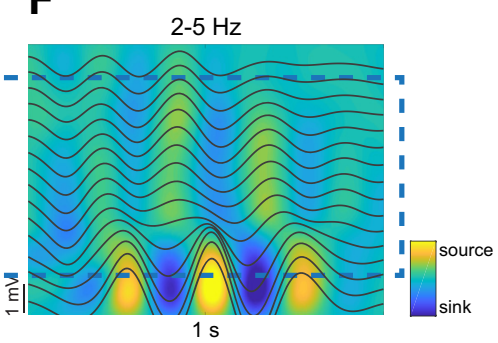

J
$\mathrm{SR}$

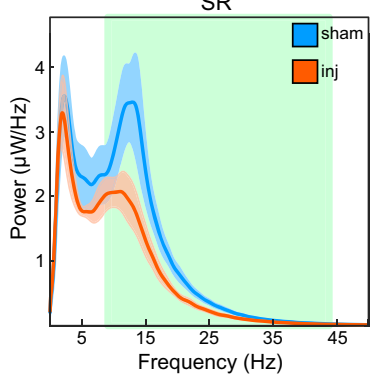

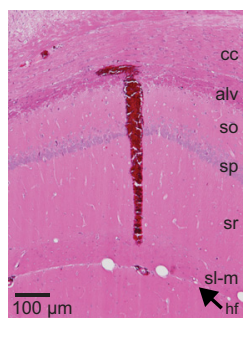

Figure 1. Laminar power profile in CA1 following TBI demonstrates attenuation of rhythmic input into stratum radiatum and reduction of high-frequency oscillations within stratum pyramidale, but preservation of anesthesia theta. $\boldsymbol{A}$, $\boldsymbol{D}$, One second example trace of wideband LFP across CA1 laminar structure in a single sham rat $(\boldsymbol{A})$ and a single injured rat $(\boldsymbol{D})$. H\&E-stained section of dorsal CA1 and drawing of a 32-channel silicon probe illustrate the approximate positions of channels relative to CA1 layers. A dominant $\sim 10-15 \mathrm{~Hz}$ oscillation in the sham is noticeably attenuated in the injured case. Arrows indicate phase reversal across SP, consistent with sharp-wave input from CA3. $\boldsymbol{B}, \boldsymbol{C}, \boldsymbol{E}, \boldsymbol{F}$, Current source density analysis of a single $500 \mathrm{~ms}$ and a separate $1 \mathrm{~s}$ LFP segment, illustrating epochs of prominent $10-15 \mathrm{~Hz}$ SR oscillations, or $2-5 \mathrm{~Hz}$ anesthesia theta oscillations, respectively, from one sham rat (B, $\boldsymbol{C})$ and one injured rat $(\boldsymbol{E}, \boldsymbol{F})$, filtered from 2 to $20 \mathrm{~Hz}(500 \mathrm{~ms}$ segment; $\boldsymbol{B}, \boldsymbol{E})$, or from 2 to $5 \mathrm{~Hz}(\boldsymbol{C}, \boldsymbol{F})$. Blue indicates a sink, while yellow indicates a source. Dark gray lines show filtered LFP. Arrows indicate rhythmic sink in SR with accompanying source in SO. Note maximal CSD sink within SR and sharp phase reversal across SP in the $\sim 10-15 \mathrm{~Hz}$ oscillation, consistent with sharp wave-like inputs, while maximal CSD sink occurs near SL-M and phase shift is gradual along the dendritic arbor from SO to SL-M in the 2-5 Hz oscillation, consistent with a theta laminar pattern. Dotted blue box indicates channels common to all animals after alignment to SP, incorporating portions of SO, SP, and SR. G, Masked heat map of significant differences (Student's $t$ test, $p<0.05$ ) in power between the sham and injured groups across 25 channels aligned to SP. Colored pixels represent a significant percentage change in power from sham to injured groups. White indicates no significant difference. A single white asterisk marks the SP channel at which the power spectrum in $\boldsymbol{F}$ is calculated. Double white asterisks mark the SR channel at which the power spectrum in $\boldsymbol{G}$ is calculated. $\boldsymbol{H}$, $\boldsymbol{I}$, Averaged power spectral densities from 50 to $700 \mathrm{~Hz}$ in SP $(\boldsymbol{H})$ and from 2 to $50 \mathrm{~Hz}$ in SR (I). Power density is expressed as log power density $(\mathrm{dB} / \mathrm{Hz})$, and frequencies are displayed on a logarithmic scale in $\boldsymbol{H}$ for better visualization. Blue lines indicate sham, orange lines indicate injured. Error bars represent the SEM. While no difference is present in the $2-5 \mathrm{~Hz}$ peak, there is a significant loss of power in the $10-15 \mathrm{~Hz}$ peak and $>120 \mathrm{~Hz}$ after injury. Green background indicates frequency ranges of significant differences in power between groups in $\boldsymbol{H}(121-700 \mathrm{~Hz})$ and $\boldsymbol{I}(10.5-43 \mathrm{~Hz}$; Student's $t$ test, $p<0.05)$. J, Representative H\&E-stained section of the dorsal hippocampus CA1 demonstrating a probe artifact with tip sitting just above SL-M. HF, hippocampal fissure.

probes at least $100 \mu \mathrm{m}$ below the deepest aligned, common channel, capturing SL-M, finding no differences in power from 2 to $5 \mathrm{~Hz}$. These data suggest that under the present levels of isoflurane anesthesia, no differences in anesthesia theta power can be observed between sham and injured animals.
While significant power differences within low frequencies were observed within SO and SR, layers containing pyramidal cell dendritic arbors, a wide band of high-frequency power loss was observed principally within the pyramidal cell layer itself (SP; Fig. 1G). Power peaks within the $\sim 100-250 \mathrm{~Hz}$ band in the sham group and a weaker 
peak in the injured group were observed within SP (Fig. $1 H$ ). Inspection of the filtered SP LFP $(90-300 \mathrm{~Hz})$ reveals brief ( $\sim 50-100 \mathrm{~ms})$ oscillatory events of increased amplitude in both groups (see Fig. 3B). However, we found a reduction in power after injury across a wide frequency range of $121-700 \mathrm{~Hz}$, with peak reduction of $74.4 \%$ power at a frequency of $231.5 \mathrm{~Hz}$ in SP (Fig. 1G,H). Highfrequency oscillations recorded within SP likely reflect local processes, whether they are coordinated spiking activity of local neurons or synaptic activity between pyramidal cells and local interneuronal networks (as occurs in ripple events; see Discussion; Schomburg et al., 2012). High-frequency power losses after injury may indicate a loss of overall local neuron firing (whether because of reduced firing rates or reduced neuron numbers), an impairment in coordination among the firing patterns of local neurons, or a loss of pyramidal cell-interneuron interactions leading, for example, to reduced ripple events (brief oscillations observed in SP within the $\sim 150-250 \mathrm{~Hz}$ band; Schomburg et al., 2012; Buzsáki, 2015). Together the power losses we observe in ipsilateral CA1 $7 \mathrm{~d}$ after TBI are frequency and anatomic layer specific, reflecting both a reduction in coordinated synaptic input into SR and a loss or discoordination of local spiking and/or synaptic activity within SP.

\section{Histopathology confirms injury severity and reveals widespread pathology of major hippocampal afferents, supporting loss of synaptic input into stratum radiatum after FPI}

A separate subset of animals (sham vs injured and without electrophysiological testing) were sacrificed at $7 d$ post-FPI for histopathological analysis to characterize the pattern and distribution of pathology within the model.

H\&E staining revealed a focal intraparenchymal lesion consistent with previous descriptions of the model (Pierce et al., 1998). Notably, the lesion was not directly below the impact site, but rather was observed laterally, affecting the temporoparietal cortex and adjacent corpus callosum. In addition to the focal lesion, regionally more diffuse injury could be observed, as evidenced by neuronal pyknosis in the hippocampus and underlying thalamus. IHC revealed axonal pathology morphologically consistent with previous descriptions in both human TBI and experimental TBI models (Gentleman et al., 1993; Johnson et al., 2013a,b, 2016; Weber et al., 2019). Specifically, abnormal APP accumulations were observed within tortuous varicose profiles and disconnected or terminal axonal bulbs secondary to transport interruption (Fig. $2 D-H$ ). In addition to the site of the focal lesion as described above, axonal pathology was observed to be more widespread throughout the brain white matter, including extensive pathology in the corpus callosum, ipsilateral thalamus, hippocampus, as well as minimal pathology in the contralateral hemisphere (Fig. 2A,B). As such, the model as described recapitulates both focal and diffuse pathologies, including cortical contusion and widespread axonal pathology, commonly observed following moderate to severe TBI in humans. In addition, the extent and distribution of pathology appears comparable to a degree of injury in the moderate to severe range for FPI (Smith et al., 1997).

Given the electrophysiological evidence of reduced coordinated synaptic input into CA1 SR, we asked whether there was structural evidence for impaired afferent projections into CA1 SR within the model. APP-positive axonal pathology was observed in all injured $(n=4)$ animals within SR and was most prominent medially (Fig. 2A,B,F,G).

However, axonal injury was not limited to SR, and APPpositive axonal swellings were consistently observed in all injured animals within the angular bundle (Fig. $2 D, E$ ) and the fimbria fornix (Fig. $2 H$ ), as well as within the SL-M of CA1 and the molecular layer of the dentate gyrus, the layers of termination of the angular bundle (via the temporoammonic and perforant pathways, respectively; Fig. $2 B$ ). Axonal pathology was minimally observed in stratum oriens, again more prominent closer to the midline.

Evidence of ongoing cell death was also observed within the CA3 subfield, a major source of excitatory projections into SR of the CA1 subfield. Specifically, in all four injured animals Fluro-Jade C-positive neurons were observed in CA3, with three of four animals also displaying immunoreactivity for Ab246 (apoptosis) in a subset of cells (Fig. $2 M, N$ ). While there was also evidence of cell death within CA1 in all injured animals (see Fig. $2 J, K$ ), this was maximal at the junction between $\mathrm{CA} 1$ and $\mathrm{CA} 2$, but absent more medially within CA1.

Together, these data suggest that FPI can result in pathologic change that may account for impaired afferent input into SR after injury. Specifically, histopathological characterization of the model suggests that this altered input may be coming from CA3 via Schaffer collaterals, whether because of CA3 pyramidal cell loss and consequently reduced CA1 presynaptic activity or because of a loss of coordination of that input.

Within the medial septum there was no evidence of any axonal pathology or Fluoro-Jade C-positive cells in any injured animals (Fig. 2S). Ab246 immunoreactivity was also absent in three injured animals, with the remaining injured animal displaying just a single positive cell (Fig. 2O$Q)$. There was similarly no evidence of overt cell death within stratum oriens.

Notably, no axonal pathology or evidence of cell death was observed in any sham animals in any regions (Fig. $2 C, I, L, R)$.

\section{FPI reduces coupling of local CA1 stratum pyramidale high-frequency activity with stratum radiatum synaptic input}

The observed post-FPI power loss of a $10-15 \mathrm{~Hz}$ oscillation in SR could reflect loss of coordinated CA3 input, as CA3 Schaffer collaterals primarily terminate in SR. Since CA3 sharp-wave input drives the occurrence of high-frequency oscillations such as ripples in CA1 SP in the nonanesthetized rat, we hypothesized that our observed reduction in SR input would exert less influence over local CA1 activity in SP, manifesting as a loss of temporal coupling between the low-frequency SR input and high-frequency oscillations in SP in addition to the observed high-frequency power loss in SP. Using the 

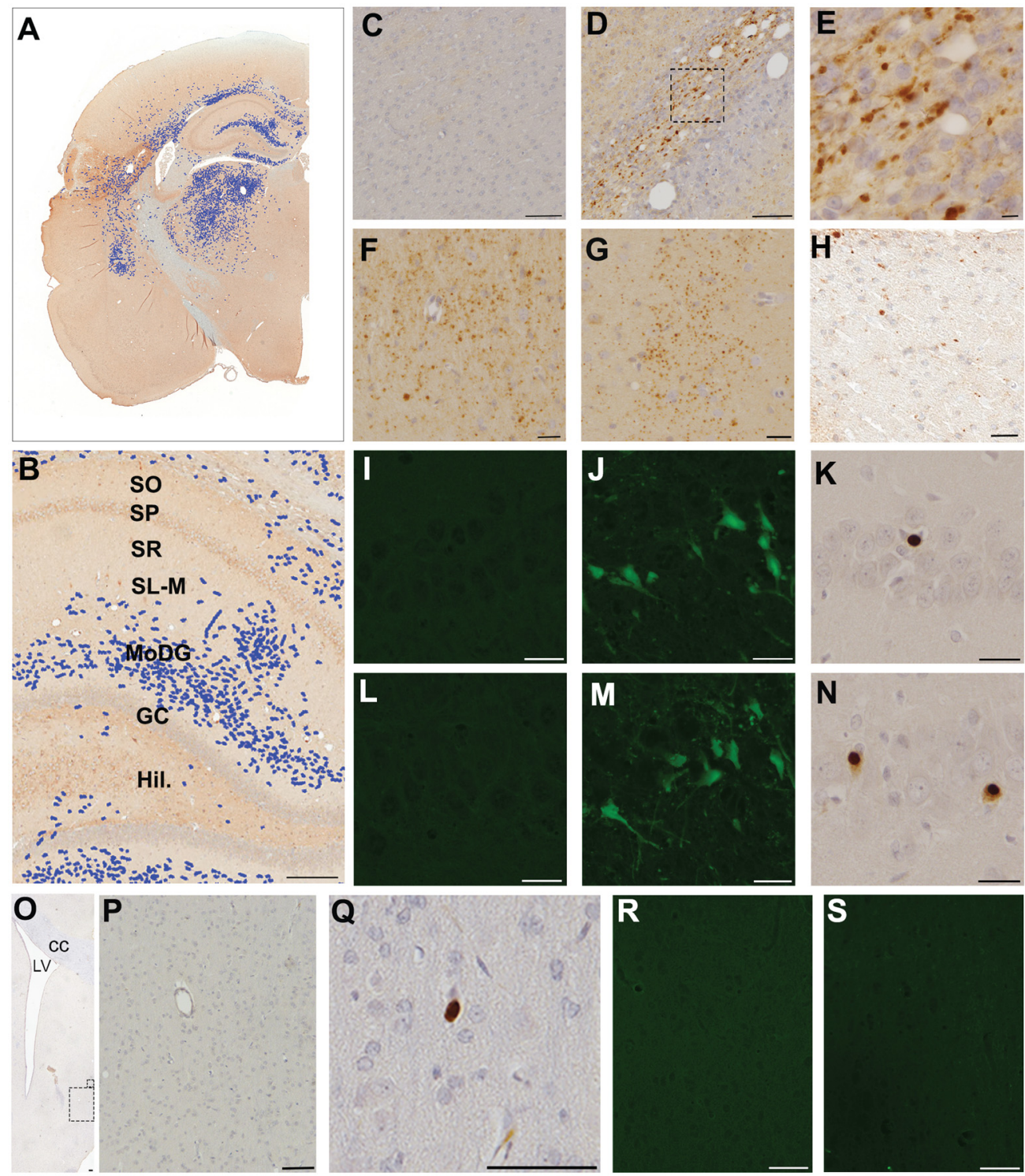

Figure 2. Neuronal and axonal injury of intrahippocampal circuitry, hippocampal afferent input pathways, and medial septum. $\boldsymbol{A}$, Whole-hemisphere map of APP-positive axonal pathology generated via manual annotation of digitally scanned APP-stained tissue section using ImageScope software (Leica Biosystems). Swollen axonal profiles were tagged (blue) to provide representative distribution map. B. Blow up of the hippocampal region identified in $\boldsymbol{A}$, illustrating APP-positive axonal pathology within SR (target of Schaffer collaterals), as well as SL-M and MoDG, targets of the angular bundle (temporoammonic and perforant pathways, respectively). $\boldsymbol{C}$, Sham animal without any APP-positive axonal pathology. $\boldsymbol{D}-\boldsymbol{H}$, Extensive axonal pathology as evidenced by pathologic APP accumulation in axonal varicosities and disconnected terminal bulbs in the angular bundle $(\boldsymbol{D})$, high-magnification image of region identified in $\boldsymbol{D}(\boldsymbol{E})$, stratum radiatum $(\boldsymbol{F}-\boldsymbol{G})$, and fimbria/fornix $(\boldsymbol{H})$ at $7 \mathrm{~d}$ post-FPI. $\boldsymbol{I}-\boldsymbol{K}$, Representative example of a sham animal (I) without any Fluoro-Jade C-positive cells in the CA1 subregion of the hippocampus. In contrast, numerous Fluoro-Jade C-positive $(\boldsymbol{J})$ and Ab246-positive $(\boldsymbol{K})$ cells indicate neuronal degeneration in CA1 of hippocampus at $7 \mathrm{~d}$ post-FPI. $\boldsymbol{L}-\boldsymbol{N}, \mathbf{S i m i l a r l y}$, an absence of Fluoro-Jade C-positive cells in the CA3 subregion of the hippocampus in a sham animal (L) versus multiple Fluoro-Jade C-positive $(\boldsymbol{M})$ and Ab246-positive $(\boldsymbol{N})$ cells in CA3 at $7 \mathrm{~d}$ post-FPI. $\mathbf{O}-\boldsymbol{Q}$, Representative Ab246 staining in the medial septum at $7 \mathrm{~d}$ postFPI. $\boldsymbol{P}$, High-magnification image of the region shown by the large box in $\boldsymbol{O}$, displaying an absence of Ab246 immunoreactivity. $\boldsymbol{Q}$, In the same case shown in $\mathbf{O}$ and $\boldsymbol{P}$, a single Ab246 cell was observed in the medial septum (high-magnification image of the small box shown in $\mathbf{O}$ ). Note this was the only Ab246-positive cell observed in the medial septum across all injured animals. $\boldsymbol{R}$, Representative image showing an absence of Fluoro-Jade $\mathrm{C}$ staining in the medial septum of a sham animal. $\boldsymbol{S}$, A similar absence of Fluoro-Jade $\mathrm{C}$ staining in the medial septum in the same injured animal/region as shown in $\boldsymbol{O}$ and $\boldsymbol{P}$. Scale bars: $\boldsymbol{B}, \mathbf{O}, \boldsymbol{P}, 100 \mu \mathrm{m}$; $\boldsymbol{C}, 10 \mu \mathrm{m} ; \boldsymbol{D}-\boldsymbol{H}, \boldsymbol{I}-\mathbf{N}, 25 \mu \mathrm{m} ; \mathbf{Q}-\mathbf{S}, 20 \mu \mathrm{m}$. MoDG, Molecular layer of dentate gyrus; GC, granule cell layer of dentate gyrus, Hil, hilus. 
spatially aligned data across all animals, we calculated the coupling of the oscillation phase over time in SR across frequencies from 8 to $20 \mathrm{~Hz}$ and the oscillation amplitude over time in SP from 1 to $300 \mathrm{~Hz}$. The strength of this coupling is quantified by the $\mathrm{Ml}$ in Figure $3 A$. Oscillatory power in the high-frequency range occurred in bursts lasting $\sim 50 \mathrm{~ms}$ (Fig. $3 B$ ). We found strong specific phase-amplitude coupling between the $\sim 11$ and $13 \mathrm{~Hz}$ phase in SR and the $\sim 150-300 \mathrm{~Hz}$ amplitude in SP in the sham group, which appeared to be severely attenuated in the injured group (Fig. $3 A$ ). In contrast, a coupling between the SR phase and amplitude of gamma-range oscillations $(30-80 \mathrm{~Hz}$; Jia and Kohn, 2011) in SP appears present in both groups. Given that the $\sim 11-13$ and $\sim 150-300 \mathrm{~Hz}$ frequency ranges fell within the frequencies of observed power drops after FPI, we directly compared the Mls between groups with a phase frequency range of $8-20 \mathrm{~Hz}$ and an amplitude frequency range of $90-300 \mathrm{~Hz}$ (Fig. $3 C$ ). We found that FPI produced a significant loss in modulation of the high-frequency amplitude in SP (190$280 \mathrm{~Hz})$ by the low-frequency phase in SR $(11-13 \mathrm{~Hz}$; Student's $t$ test, $p<0.05$; Fig. 3D). Further, the maximum amplitude in the high-frequency signal aligned closely with the phase of maximal downward deflection in the low-frequency signal, which corresponds to the phase of maximal current influx into SR (Fig. $3 D$ )-a relationship expected if the low-frequency signal reflects SR synaptic input driving local neuronal activity in SP (Buzsáki, 2015). In contrast, the coupling between SR phase and SP mid-gamma oscillatory amplitude was not statistically different (Student's $t$ test, $p>0.05$ ). These results suggest that local neuronal activity in SP (observed as high-frequency oscillations) has been partially decoupled from the observed oscillatory input into SR after FPI. This may be due directly to loss of that input, disrupted coordination among the afferent neurons, or a reduced ability of local CA1 neurons/networks to properly respond to that input.

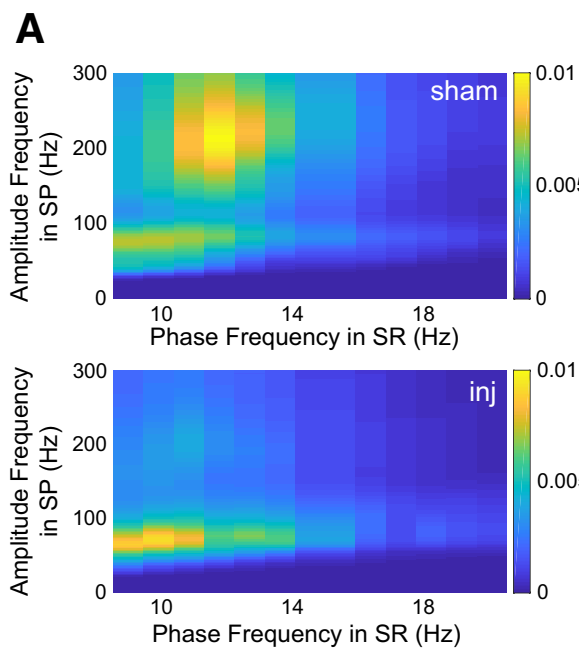

B

C
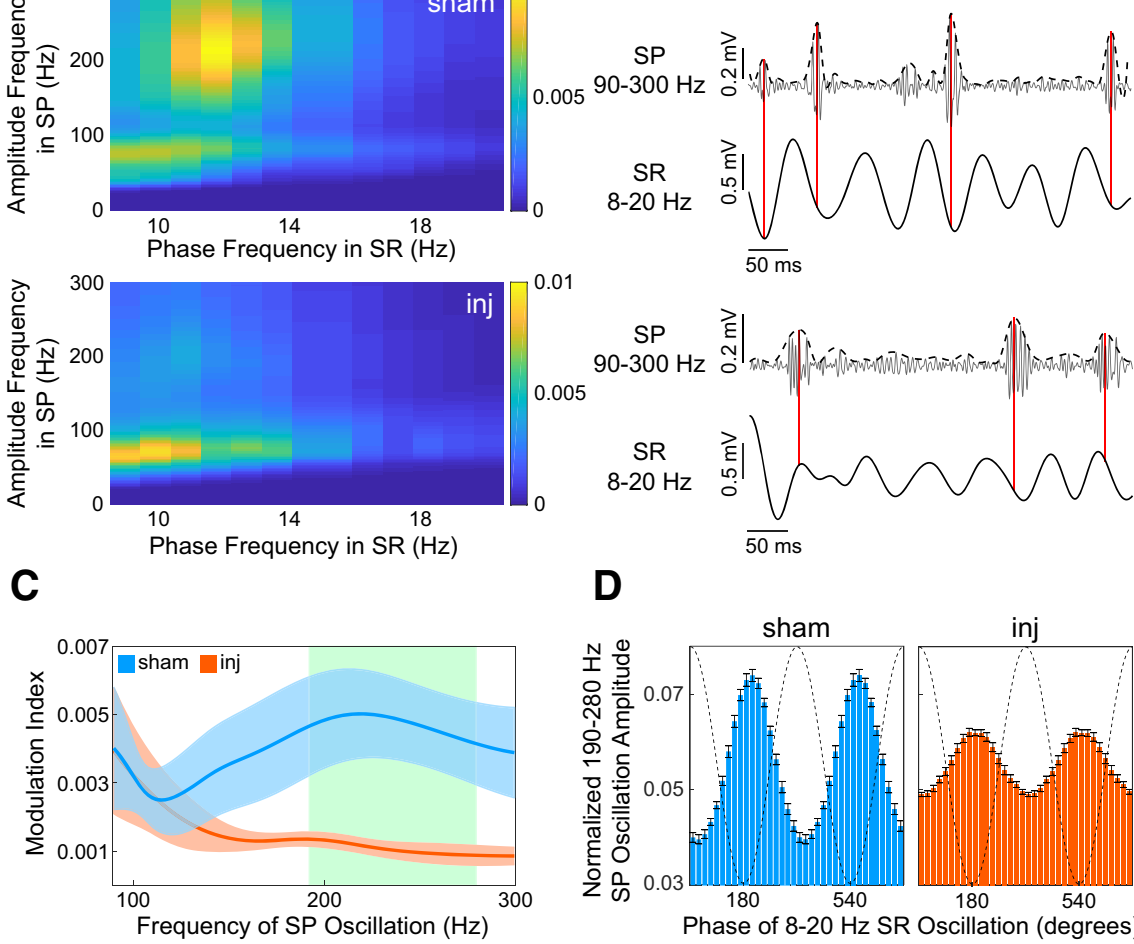

D

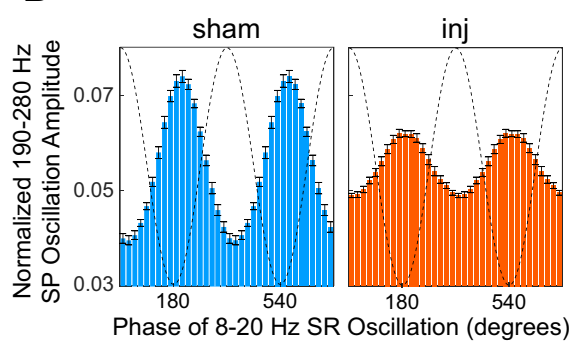

Figure 3. TBI reduces phase-amplitude coupling between rhythmic stratum radiatum input and high-frequency oscillations in stratum pyramidale. A, Mean sham (top) and injured (bottom) comodulograms depicting the modulation index of phase frequency (8$20 \mathrm{~Hz})$ in SR with amplitude frequency $(0-300 \mathrm{~Hz})$ in SP. Warmer colors indicate higher modulation indices. Note the strong coupling between the $\sim 10-15 \mathrm{~Hz}$ phase in SR and high-frequency oscillation ( $200-300 \mathrm{~Hz})$ amplitude in the sham group, which is lost after injury. B, A 500 ms CA1 tracing from a single sham rat (top) and an injured rat (bottom), demonstrating phase-amplitude coupling between dominant SR rhythmic input and high-frequency oscillations in SP. Top tracing is the normalized bandpass filtered LFP $(90-300 \mathrm{~Hz})$ in SP. The dotted line is a smoothed envelope for visual clarity. Bottom tracing is the simultaneous bandpass filtered LFP $(8-20 \mathrm{~Hz})$ in SR. Vertical red lines highlight alignment of high-frequency oscillatory peaks to a consistent phase of the low-frequency input into SR in the sham group. This alignment becomes inconsistent in the injured group. $\boldsymbol{C}$, Mean ( \pm SEM) modulation index of the $8-20 \mathrm{~Hz}$ SR input as a function of high-frequency oscillation amplitude in SP. Green background indicates a high-frequency band $(190-280 \mathrm{~Hz}$ ) of significant loss of modulation by the SR input after injury. $\boldsymbol{D}$, Mean ( \pm SEM) phase-amplitude coupling between the 8 and $20 \mathrm{~Hz}$ phase in SR and the 190-280 Hz amplitude in SP. Note the maximum high-frequency amplitude in SP just follows the minimum low-frequency phase, which corresponds to the phase of maximum inward current in SR. Dotted lines represent the phase of the $8-20 \mathrm{~Hz}$ oscillation. Blue and orange represent the sham and injured groups, respectively, in $\boldsymbol{C}$ and $\boldsymbol{D}$. 


\section{Local CA1 neurons maintain normal firing rate capacity after FPI}

One possible explanation of the observed high-frequency power losses within SP after FPI could be reduced CA1 neuron firing rates, reflecting an impaired ability to achieve a normal response to synaptic input. To address this possibility, we explored whether the firing properties of isolated CA1 neurons were altered after injury. We isolated single units from the raw signal and then classified them into putative pyramidal cells and putative interneurons (Fig. 4B). Using this scheme, we were able to classify 78 of $116(67 \%)$ sham units and 59 of $86(69 \%)$ injured units. We isolated a total of 49 putative pyramidal cells and 34 putative interneurons in the sham group and 34 putative pyramidal cells and 25 putative interneurons in the injured group. We found no significant difference in the median number of putative pyramidal cells per rat [sham group, 8 (range, 8-16); vs injured group, 6 (range, 2-12); Mann-Whitney test, $p=0.33$; Fig. $4 D$, top], nor in the median number of putative interneurons per rat in the injured group [sham group, 6 (range, 4-8); vs injured group, 4 (range, 3-7); Mann-Whitney test, $p=0.58$; Fig. $4 D$, bottom]. We further found no differences in putative interneuron numbers within each anatomic layer (SO, $p=0.80$; SP, $p=0.12$; SR, $p=0.51$; Fig. 4G). Strikingly, the mean firing rates were also unchanged after injury for putative pyramidal cells (sham group, $1.25 \pm 0.91 \mathrm{~Hz}$; vs injured group, $1.26 \pm 0.91 \mathrm{~Hz}$; Welch's $t$ test, $p=0.98$ ), putative interneurons (sham group, $5.12 \pm 7.50 \mathrm{~Hz}$; vs injured group, $4.26 \pm 7.51 \mathrm{~Hz}$; Welch's $t$ test, $p=0.68$ ), and putative interneurons within each layer separately (SO, $p=0.09$; SP, $p=0.89$; SR, $p=0.13$ ), demonstrating an overall preservation of firing rates between sham and injured animals (Fig. 4D).

\section{Injured neurons lose proper entrainment to rhythmic stratum radiatum input and local high-frequency oscillations}

An important presumed function of oscillatory synaptic input in the hippocampus is to precisely organize the firing patterns of local neurons, leading to neurons firing at a particular phase relative to an oscillation or shifting that phase in a predictable fashion over time for encoding purposes (O'Keefe and Recce, 1993). Although we found that overall neuron firing rates remained stable among isolated single units after FPI, it is possible that they are unable to appropriately phase lock to organizing oscillatory activity. To directly address whether CA1 neuron firing patterns are influenced by the observed oscillatory SR input and assess whether this influence is impaired after injury, we investigated the phase relationship of spiking patterns of isolated single neurons in CA1 to the observed $\sim 10$ $15 \mathrm{~Hz}$ oscillatory input in SR (so-called "entrainment"; Fig. 5); 48 of 49 (98\%) putative pyramidal cells were significantly entrained in the sham group, while only 27 of 34 (79\%) cells were entrained in the injured group, a drop in number of nearly $20 \%$ ( $\chi^{2}$ test, $\left.p<0.0001\right)$. In contrast, the number of putative interneurons entrained was similar in sham and injured animals: 27 of 29 (93\%) putative interneurons in the sham group, and 22 of $25(88 \%)$ in the injured group ( $\chi^{2}$ test, $\left.p=0.31\right)$.
To assess the strength of entrainment of individual neurons to this oscillation, we used the resultant vector, which is a measure of how tightly distributed the phases of each action potential in the spike train of a given neuron are around the mean phase of all action potentials (a resultant vector of 1 indicates that all spike phases are identical and the neuron is strongly entrained, while a value of 0 indicates that there is no preferred phase and no entrainment). We found a significant drop in the mean resultant vector, or strength of entrainment, of putative pyramidal cells (sham group, $0.24 \pm 0.09$; injured group, $0.19 \pm 0.07$; Welch's $t$ test, $p=0.012$ ), but not interneurons (sham group, $0.27 \pm 0.13$; injured group, $0.22 \pm 0.10$; Welch's $t$ test, $p=0.13$ ) after injury (Fig. $5 A, B$ ).

We then asked how well neurons as a group were entraining to the proper phase after injury. Surprisingly, putative CA1 pyramidal cells in the injured group were unable to maintain a consistent phase preference relative to the SR oscillatory input compared with those in the sham group, displaying both a significantly wider variability in their preferred phase of entrainment (Kuiper test, $p<0.05$; Fig. $5 A$, top), as well as an altered mean phase of entrainment (sham group, $193.8 \pm 24.9^{\circ}$; injured group, $208.7 \pm 32.7^{\circ}$; Watson-Williams test, $p=0.039$; Fig. $5 A$, top). Visual inspection of the circular distributions in Figure $5 A$ strongly suggests a secondary preferred phase of $\sim 240^{\circ}$ in the injured group, but not in the sham group. There were no differences in the interneuron mean preferred phase (sham group, $181.8 \pm 35.6^{\circ}$; injured group, $189.0 \pm 50.0^{\circ}$; Watson-Williams test, $p=0.61$ ) or in the wide distribution of preferred phases between groups (Kuiper test, $p=0.13$; Fig. 5B, top).

We also examined CA1 neuron entrainment to SP highfrequency oscillations, revealing that it had become less focused in the pyramidal cells (Fig. 5C). In sharp contrast to SR oscillation entrainment deficits after injury, there were significant increases in the entrainment of putative pyramidal cells to the $55-175$ and $280-400 \mathrm{~Hz}$ oscillatory activity in SP (Student's $t$ test, $p<0.05$; Fig. $5 C$ ), broadening the peak of entrainment across a wider range of high-frequency oscillations after injury. Interneuron entrainment in SP was highly variable and was not altered following FPI (Fig. 5D).

These results indicate that CA1 pyramidal cells and interneurons are less able to synchronize to oscillatory SR synaptic input after FPI, whether because of decreased synaptic input itself, or because of an altered response, possibly contributing to the broad high-frequency power losses observed in SP after FPI. More importantly, loss of the precise timing relationship between CA1 synaptic inputs and CA1 pyramidal cell firing patterns could have significant implications for the ability of the hippocampus to encode information.

\section{TBI leads to a reduction in coordinated rhythmic inhibition of medial septal neurons by CA1}

The medial septum is thought to play an important role in the generation and maintenance of hippocampal theta oscillations. Conversely, during periods when CA1 activity is dominated by sharp-wave ripples, feedback projections to the MS have been shown to inhibit MS cell firing (Dragoi et al., 
A

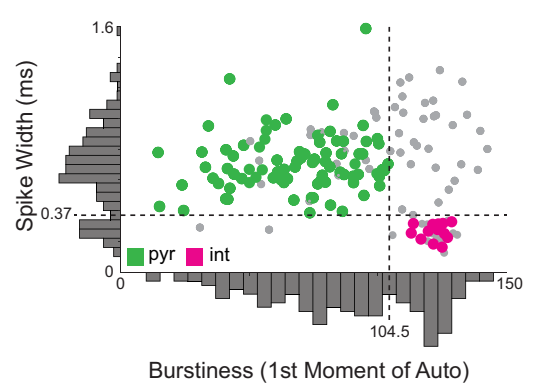

C
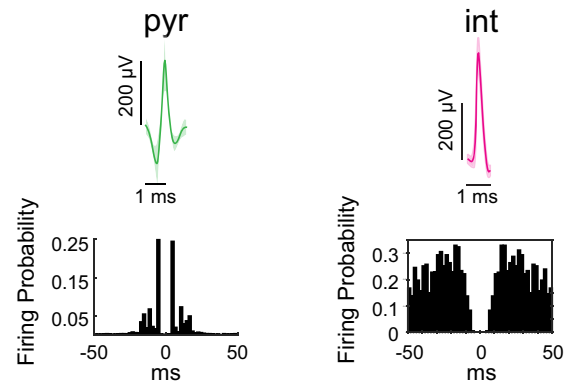

B

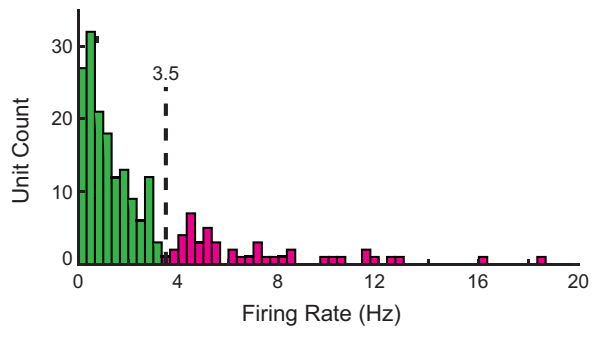

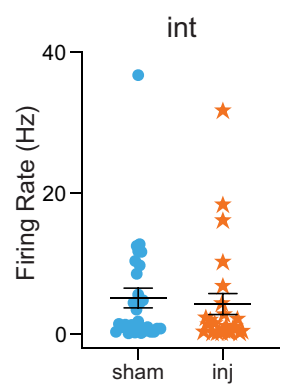

E
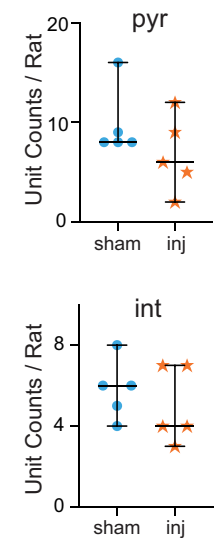

$\mathbf{F}$
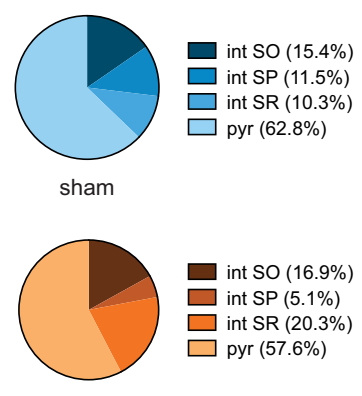

inj

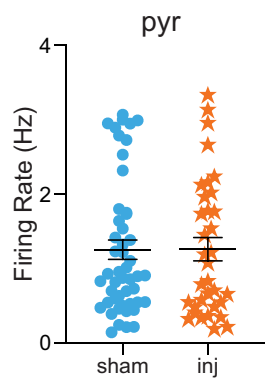

G

H

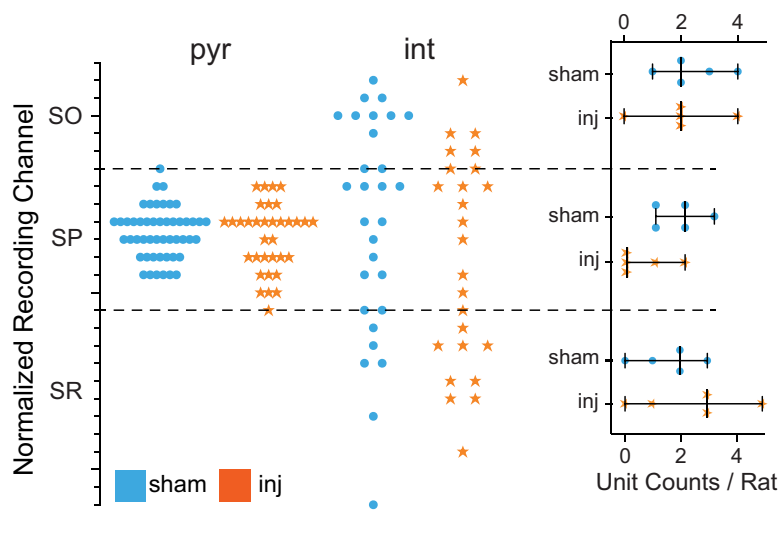

Figure 4. Neuronal firing rates and putative neuron number are preserved in CA1 following TBI. $\boldsymbol{A}, \boldsymbol{B}$, Division of isolated CA1 single units within SP into putative pyramidal cells and putative interneurons. $\boldsymbol{A}$, Dark gray bars indicate histograms of the first moment of the autocorrelogram (x-axis), with lower numbers indicating higher burstiness, and spike width (at $25 \%$ maximum height) of the unfiltered waveforms ( $y$-axis). B, Firing rate histogram of all isolated single units. Each feature reveals two distributions of single units, defining the following two clusters: magenta circles in the right bottom quadrant (narrow, tonic, fast spiking cells-putative interneurons); and green circles in the left top quadrant (wide, bursting, slower-spiking cells-putative pyramidal cells). Gray circles represent units that could not be subtyped. C. Example of single isolated putative pyramidal cell (left) and putative interneuron (right). Top, Mean $( \pm S D$ ) waveforms. Bottom, Autocorrelograms expressed as firing probability as a function of time bin (bin size, 2 ms). Note in the putative pyramidal cell the wider waveform as well as the multiple short-latency peaks in the autocorrelogram that correspond to burst activity compared with the putative interneuron. $\boldsymbol{D}$, Overall mean ( $\pm \mathrm{SEM}$ ) firing rates of putative pyramidal cells (left) and interneurons (right) demonstrating no change in baseline activity between sham and injured animals. $\boldsymbol{E}$, No change in the median (with $95 \% \mathrm{Cl}$ ) number of isolated putative pyramidal cells (top) or interneurons (bottom) per rat after injury. $\boldsymbol{F}$, Pie charts depict the relative distribution of putative neuronal subtypes across CA1 layers. $\mathbf{G}$, Laminar distribution of all isolated putative pyramidal cells (circles) and interneurons (stars) as a function of recording channel normalized to SP. Dotted lines indicate SO-SP and SP-SR borders. $\boldsymbol{H}$, Plots compare median (with $95 \% \mathrm{Cl}$ ) number of isolated putative interneurons per rat between groups in each layer. There is no significant dropout of putative interneurons within any layer. Blue and orange represent sham and injured rats, respectively. Circles and stars represent putative pyramidal cells and interneurons, respectively. inj, Injured; pyr, pyramidals; int, interneurons.

1999). We would therefore expect synchronization of MS neuron firing with both CA1 theta oscillations and sharpwave ripples. Thus, if our observed oscillation in SR under anesthesia indeed reflects rhythmic sharp waves, we would expect MS neurons to be rhythmically inhibited by it. Given reports of reduced theta power after TBI as well as our observed reduction in SR synaptic input after FPI, we asked whether there was either reduced 
A

B int
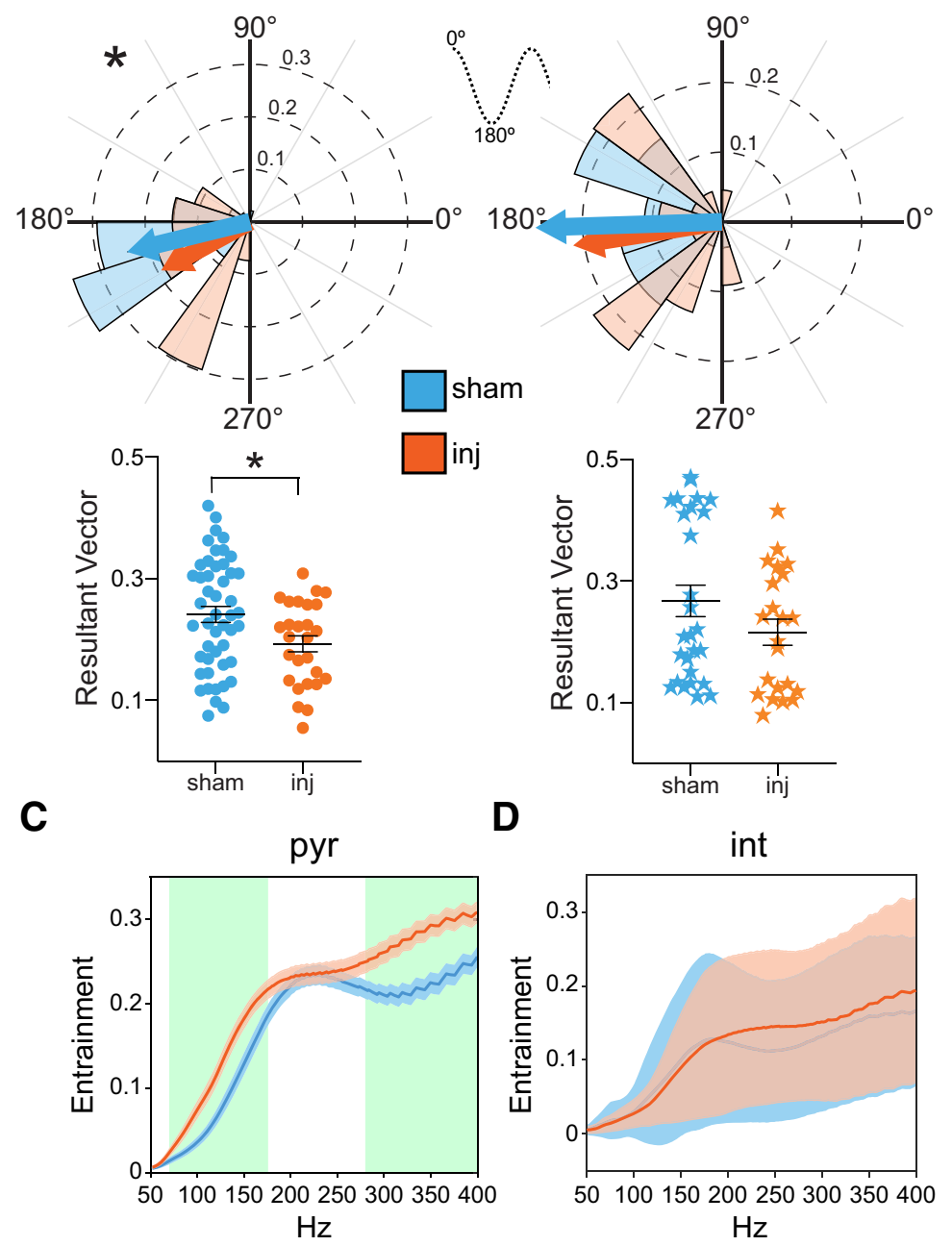

Figure 5. Despite normal firing rates, CA1 neuronal entrainment to rhythmic stratum radiatum input and local high-frequency oscillations is altered following injury. $\boldsymbol{A}, \boldsymbol{B}$, Entrainment of single neurons to rhythmic SR oscillation (8-20 Hz). Top, Polar histogram depicting preferred phase of entrainment of putative CA1 pyramidal cells $(\boldsymbol{A})$ or interneurons $(\boldsymbol{B})$ that are significantly entrained to 8$20 \mathrm{~Hz}$ oscillation in SR. There is a significantly broader distribution of preferred phase angles among the injured pyramidal cells as well as a subset that appears to entrain with $\mathrm{a} \sim 30^{\circ}$ offset. Solid arrow vectors indicate the mean phase angle of entrainment with length equal to the mean resultant vector. Asterisk indicates significant difference in distribution $(p<0.05)$. Dotted sinusoidal wave illustrates phase angle of LFP signal. Bottom, Comparison of the group mean ( \pm SEM) resultant vector lengths for each entrained putative pyramidal cell $(\boldsymbol{A})$ or interneuron $(\boldsymbol{B})$. Resultant vector indicates the variance of individual spike phases about the mean for a given single putative neuron (resultant vector of 1 indicates identical phases for all spikes), giving a measure of the degree of entrainment of a given putative neuron. The degree of entrainment of the pyramidal neurons was significantly lower following injury, suggesting a decrease in the ability of these neurons to follow the oscillation. $\boldsymbol{C}, \boldsymbol{D}$, Degree of entrainment (mean \pm SEM) of significantly entrained putative pyramidal neurons $(\boldsymbol{C})$ and interneurons $(\boldsymbol{D})$ to local high-frequency oscillations $(50-400 \mathrm{~Hz})$ as a function of frequency. A focal peak frequency band $(\sim 200-250 \mathrm{~Hz})$ in sham putative pyramidal cell entrainment becomes a broader frequency range of increased entrainment after injury, incorporating higher and lower frequencies. Green background indicates frequencies of significant difference between groups $(p<0.05)$. Blue and orange represent sham and injured, respectively.

synchronization of MS neuron firing with CA1 anesthesia theta or a reduction in the modulation of the MS by CA1 SR oscillatory input (Fig. 6).

A total of 147 and 182 medial septal cells were isolated in the sham and injured groups, respectively [sham group, 25 (range, 15-50) cells per animal; vs injured group, 33 (range, 17-54) cells per animal; Mann-Whitney, $p=0.45$ ). As different neuron subtypes within the MS are known to play different roles in the regulation of hippocampal activity, we used a two-step process consisting of established waveform shape criteria adapted from King et al. (1998) followed by a firing rate cutoff to divide the isolated MS cells into putative subtypes: cholinergic versus GABA/Glu populations (Fig. 6A-E). Examples of putative GABA/Glu and putative cholinergic cells are shown in Figure $6 A ; 82$ putative GABA/Glu cells were isolated in the sham group 
A

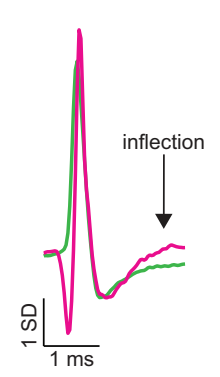

G

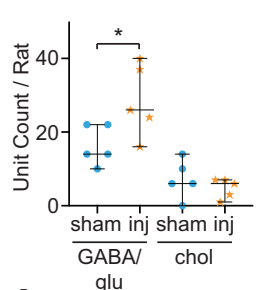

$\mathbf{L}_{\times 10^{-2}}^{\text {glu }}$ sham

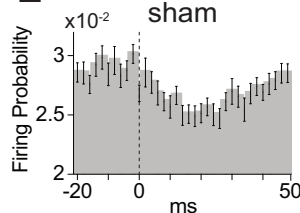

B
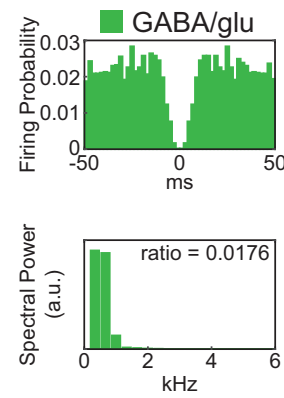

H

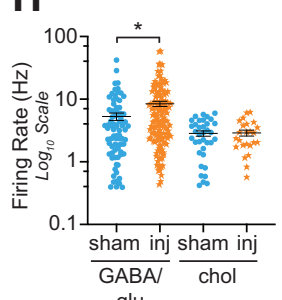

glu

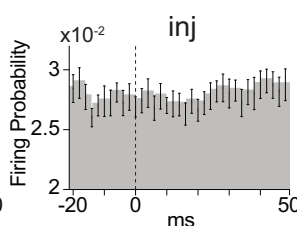

C
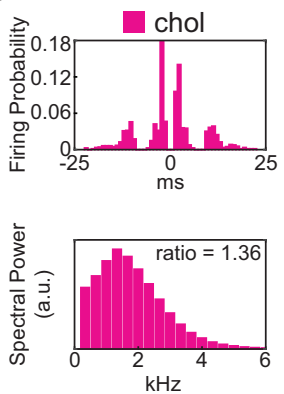

I

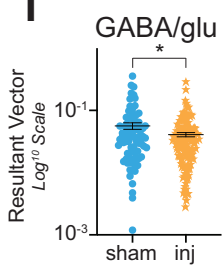

chol

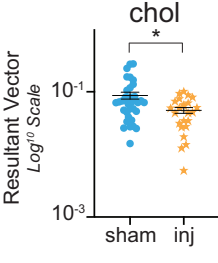

D

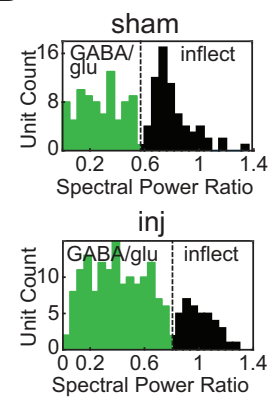

E
J
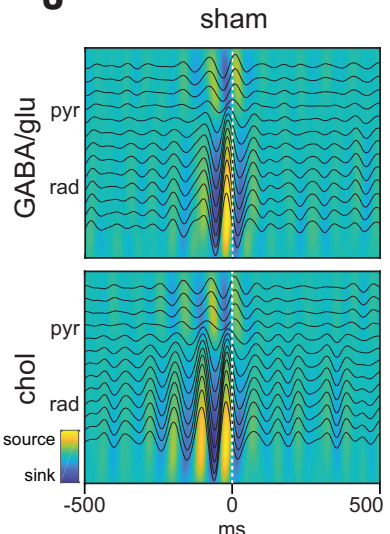

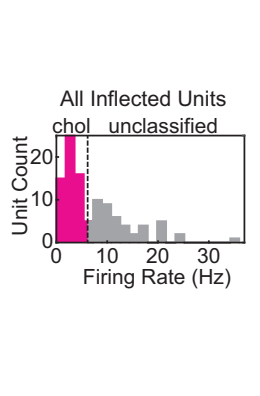

$\mathbf{F}$

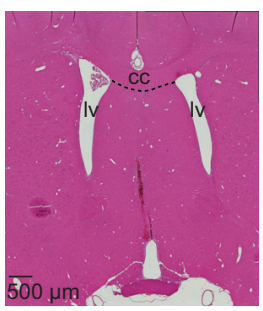

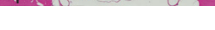

Figure 6. TBI leads to a reduction in rhythmic CA1 inhibition of medial septum. $\boldsymbol{A}$, Unfiltered averaged waveforms of isolated MS sham putative GABAergic/glutamatergic (magenta) and putative cholinergic (green) single units, amplitude normalized to highlight waveform shape differences. Note "inflection" during repolarization, characteristic of cholinergic neurons. $\boldsymbol{B}$, $\boldsymbol{C}$, Top, Autocorrelograms of same single MS units. Bottom, Power spectrum (1-16 kHz, 25 bins) of same single MS units. Spectral power ratio is of fourth to second frequency bins. Putative cholinergic waveform inflection manifests as increased power at higher frequencies. $\boldsymbol{D}$, Histograms of spectral power ratios for all sham (top) and injured (bottom) MS units. Two distributions are apparent, defining putative GABAergic/glutamatergic and cholinergic populations. $\boldsymbol{E}$, Firing rate histogram of all putative single MS units meeting spectral ratio criteria as "inflected" units, revealing a distinct subpopulation of low-firing units, which we have classified as putative cholinergic units. $\boldsymbol{F}$, Representative H\&E-stained section of the medial septum with tetrode artifact. $\boldsymbol{G}$, Increased number of median (with $95 \% \mathrm{Cl}$ ) isolated putative GABAergic/glutamatergic units per rat after injury, but no change in putative number of cholinergic units. $\boldsymbol{H}$, Increased mean firing rate of isolated putative GABAergic/glutamatergic units after injury, but no change in putative cholinergic unit firing rates, suggesting increased excitation or loss of inhibition. $\mathbf{I} \boldsymbol{K}$, Modulation of isolated MS units by $8-20 \mathrm{~Hz}$ oscillation in SR of CA1. I, Reduction in the mean strength of MS unit modulation (resultant vector) by CA1 8-20 Hz in putative GABAergic/ glutamatergic (top) and cholinergic (bottom) subgroups. J, MS unit spike-triggered average of CA1 8-20 Hz filtered LFP, averaged across all putative GABAergic/glutamatergic (top) and cholinergic (bottom) MS units significantly modulated by the CA1 oscillation in the sham (left) and injured (right) groups. Injured color maps normalized to sham color maps. Note reduction in $\sim 10-15 \mathrm{~Hz} C A 1 \mathrm{SR}$ oscillation just preceding the MS action potential (dotted white line). $\boldsymbol{K}$, Reduction in the peak positive to the peak negative voltage difference in the averaged spike-triggered averages of the deepest channel in stratum radiatum depicted in $\boldsymbol{J}$ for both putative GABAergic/ glutamatergic and cholinergic MS units. $L$, Averaged peristimulus time histograms for all putative MS units (GABAergic/glutamatergic and cholinergic) significantly modulated by CA1 8-20 Hz SR oscillation, triggered off the trough of that oscillation (dotted lines) in the sham (left) and injured (right) groups. As the maximal unit firing probability in CA1 occurs near the 8-20 Hz filtered SR trough (Fig. 5), the lowest MS unit firing probability occurring just after this trough in the sham group, but not in the injured group, suggests that coordinated rhythmic inhibition of MS by CA1 is diminished after injury. Single asterisks indicate a significant difference $(p<0.05)$.

[median per rat: 14 (range, 10-22), while 143 were isolated in the injured group (median per rat: 26 (range, 16-40)], representing a significant increase in the number per rat (Fig. 6G; Mann-Whitney test, $p=0.032$ ). Thirty-six putative cholinergic were isolated in the sham group [median per rat: 6 (range, 0-14)] and 24 in the injured group [median per rat: 6 (range, 1-7)], which did not differ significantly between groups (Fig. 6G; Mann-Whitney test, $p=0.63$ ). Similarly, the mean firing rate of putative GABA/ Glu cells increased after injury (sham group, $5.30 \pm$
$6.42 \mathrm{~Hz}$; vs injured group, $8.46 \pm 9.76 \mathrm{~Hz}$; Welch's $t$ test, $p=0.0039$ ), while that of putative cholinergic cells did not (sham group, $2.84 \pm 1.64 \mathrm{~Hz}$; vs injured group, $2.89 \pm$ $1.55 \mathrm{~Hz}$; Welch's $t$ test, $p=0.92$; Fig. $6 H$ ). These findings suggest a selective disinhibition of MS GABA/Glu neurons after injury.

To assess the interactions between MS neuron firing and either anesthesia theta or the observed $\sim 10-15 \mathrm{~Hz}$ oscillatory synaptic input into CA1 SR, we first assessed the phase relationship between the trough of each 
oscillation and the spiking patterns of individual isolated MS neurons. Proportions of entrained cells after injury were preserved. Among putative GABA/Glu cells, we found that 24 of $82(29 \%)$ in the sham group and 52 of $143(36 \%)$ in the injured group were entrained to anesthesia theta (no difference: $\chi^{2}$ test, $p=0.06$ ), while 28 of 82 $(34 \%)$ in the sham group and 44 of $143(31 \%)$ in the injured group were entrained to the CA1 $10-15 \mathrm{~Hz}$ SR input (no difference: $\chi^{2}$ test, $p=0.73$ ). Among putative cholinergic cells, we found 18 of $36(29 \%)$ in the sham group and 11 of $24(46 \%)$ in the injured group were entrained to anesthesia theta (no difference: $\chi^{2}$ test, $p=0.68$ ), while 18 of $36(50 \%)$ in the sham group and 9 of $24(38 \%)$ in the injured group were entrained to the CA1 $10-15 \mathrm{~Hz}$ SR input (no difference: $\chi^{2}$ test, $p=0.22$ ). Among those putative cells that were significantly entrained, we measured the strength of that entrainment (mean resultant vector), finding no differences among putative GABA/Glu cells (sham group, $0.10 \pm 0.09$; vs injured group, $0.08 \pm 0.06$; Welch's $t$ test, $p=0.22$ ), or among putative cholinergic cells (sham group, $0.11 \pm 0.06$; vs injured group, $0.12 \pm$ 0.07 ; Welch's $t$ test, $p=0.75$ ) to anesthesia theta. However, both putative cell subtypes lost strength of entrainment to the CA1 $10-15 \mathrm{~Hz}$ SR input after injury (GABA/Glu: sham group, $0.06 \pm 0.06$; vs injured group, $0.04 \pm 0.04$; Welch's $t$ test, $p=0.04$; cholinergic: sham group, $0.09 \pm 0.07$; vs injured group, $0.05 \pm 0.03$; Welch's $t$ test, $p=0.005$; Fig. $6 /$ ). These results suggest that MS neurons are indeed synchronized both to CA1 anesthesia theta oscillations and sharp wave-like oscillations, but while anesthesia theta synchronization appears preserved after injury, sharp wave-like oscillation synchronization is impaired.

If the observed $\sim 10-15 \mathrm{~Hz}$ SR oscillation in CA1 indeed represents rhythmic sharp wave-like input from CA3, we would expect the oscillation to drive MS neuron firing patterns and not the reverse. To test this, we computed the MS spike-triggered average of the CA1 LFP for each significantly entrained putative MS cell. The averaged spiketriggered averages for both the putative GABA/Glu and cholinergic cell subgroups revealed a robust $\sim 11 \mathrm{~Hz}$ oscillation with the expected maximal amplitude and CSD sink within SR and a sharp phase reversal from SO to SR just before the MS neuron action potential in the sham group. This oscillation was severely attenuated in the injured group (Fig. 6J). To quantify this, we measured the difference between the maximum positive voltage deflection and the maximum negative deflection in SR of the averaged spike-triggered averages, confirming a significant reduction in the $\sim 11 \mathrm{~Hz}$ oscillation occurring just before the action potential in both GABA/Glu (sham group, $162.0 \pm 146.6 \mu \mathrm{V}$; vs injured group, $95.6 \pm 114.1 \mu \mathrm{V}$; Welch's $t$ test, $p=0.048$, Fig. $6 K$, top) and cholinergic (sham group, $222.7 \pm 174.5 \mu \mathrm{V}$; vs injured group, $87.1 \pm$ $51.0 \mu \mathrm{V}$; Welch's $t$ test, $p=0.0059$; Fig. $6 K$, bottom) MS cells after injury. These results suggest a loss of modulation of MS neuron firing patterns after injury by CA1 feedback driven by the observed CA1 SR oscillation.

As the projections from CA1 to MS are known to inhibit the firing of MS neurons time locked to the maximal coordinated CA3 input into CA1 (maximum negative deflection in the sharp-wave LFP; Dragoi et al., 1999), we explored whether our observed modulation of MS neuron firing patterns by $\sim 10-15 \mathrm{~Hz}$ oscillatory CA1 SR input was inhibitory. We calculated the average PSTH in each group for all MS neurons significantly modulated by the observed $10-15 \mathrm{~Hz}$ CA1 SR input triggered off the trough of that oscillation. In the sham group, this revealed a significant inhibition of the average MS neuron firing probability $\sim 14 \mathrm{~ms}$ after the maximal negative deflection (trough) of the CA1 SR oscillation (Fig. 6L, left). This inhibitory influence was not detectable in the injured group PSTH (Fig. 6L, right).

Together, these data suggest that while the MS maintains synchronization with CA1 anesthesia theta, it may lose coordinated inhibition from rhythmic CA1 sharp wave-like oscillations after injury. Increased numbers of detected putative GABA/Glu (but not cholinergic) cells as well as their increased firing rates after injury suggest they may be preferentially disinhibited. Lack of observed MS structural pathology suggests these changes may be a consequence of reduced or dyscoordinated hippocamposeptal projections into MS, rather than an injured MS network.

\section{Discussion}

TBI can lead to profound cognitive dysfunction both acutely and chronically following even mild injury (Hoge et al., 2008; Monti et al., 2013; Maas et al., 2017; Wilson et al., 2017). The hippocampus is often identified as a potential substrate for this disruption as well as a potential target for neuromodulatory treatment because of its prevalent role in cognition and memory, and its demonstrated vulnerability in TBI (Santhakumar et al., 2001). Explanations for hippocampal-dependent dysfunction range from synaptic imbalance (Howard et al., 2007) to changes in coding and firing properties in remaining neurons in the hippocampal network (Wolf and Koch, 2016), such as reduced spatial specificity of place fields (Broussard et al., 2020). Several studies in the intact rodent brain after TBI have shown theta band specificpower losses in the hippocampus (Fedor et al., 2010; Lee et al., 2013, 2015), an intriguing finding given that theta oscillations are thought to play a central role in memory encoding (McNaughton et al., 2006a,b). However, Paterno et al. (2016) have reported only broadband power losses in the hippocampus that offer an alternate explanation for theta decreases, postulating that broadband power reflects either the magnitude or synchrony of local multiunit activity. An important question is therefore whether oscillatory power losses in the intact brain after TBI are anatomic layer specific and frequency band specific, and therefore a reflection of changes in distinct synaptic inputs and/or local firing properties, as well as whether the modulation of single-neuron firing patterns by these oscillations is impaired. At $7 \mathrm{~d}$ following TBI, we found the following: (1) that CA1 power changes after TBI are anatomic layer specific and frequency band specific, revealing that low-frequency power losses under anesthesia are localized to the dendrites in SR, potentially 
reflecting reduced oscillatory input from CA3, while highfrequency power losses are confined to the perisomatic region, likely reflecting altered firing patterns and local interneuronal activity in response to synaptic input; (2) that theta oscillation power is unchanged under deep isoflurane anesthesia; (3) that isolated single CA1 neurons are able to maintain normal firing rates despite reduced oscillatory input to SR, yet are unable to maintain proper synchronization to that input; and (4) that coordinated inhibition of isolated single MS neurons by the observed dominant CA1 SR oscillation is impaired after injury, potentially reflecting a loss of inhibitory feedback from CA1 to the MS during sharp-wave ripples.

\section{TBI leads to loss of CA1 synaptic input}

The SR of CA1 is the principle target of CA3 pyramidal cell projections via the Schaffer collaterals. We found a loss of low-frequency oscillatory power confined to SR, consistent with our observation of CA3 neurodegeneration and Schaffer collateral axonal pathology $7 \mathrm{~d}$ after injury. During consummatory behaviors and non-rapid eye movement (REM) sleep, episodes of synchronized input into SR from CA3, termed sharp waves, organize local CA1 neuronal activity into bursts, a process thought to be important for memory decoding (Buzsáki, 1986, 2015). Synchronized input into SR from CA3 has been observed under deep isoflurane anesthesia in the form an $\sim 10$ $15 \mathrm{~Hz}$ oscillation with the same anatomic pattern in the CA1 LFP and with the same synchronization of CA1 neuronal activity, as observed with sharp-wave events in the nonanesthetized rat. Further, $2-5 \mathrm{~Hz}$ oscillations that follow the anatomic pattern of awake theta oscillations (here termed anesthesia theta) can be observed under light isoflurane anesthesia (Lustig et al., 2016). Whether this pattern is the equivalent of theta observed during exploratory behavior or, for example, REM sleep in terms of the driving inputs that shape it, requires further study.

We found $\sim 10-15 \mathrm{~Hz}$ rhythmic SR input dominated the CA1 LFP in our anesthesia recordings in both groups, but was significantly reduced in power after TBI, while the brief episodes of anesthesia theta LFP patterns were unchanged. Loss of CA3 neurons and accompanying alterations in CA3-CA1 synaptic activity are a core feature of the lateral FPI model (Smith et al., 1991, 1997; Hicks et al., 1993; Grady et al., 2003), as well as other TBI models (Baldwin et al., 1997; Scheff et al., 2005; Norris and Scheff, 2009; Wolf et al., 2017). Thus, a loss in SR synaptic input after TBI could very well reflect reduced, or disrupted, CA3 inputs via Schaffer collaterals. The present study provides the first in vivo link between the known loss of CA3 activity after FPI and oscillatory power changes in CA1 in the intact brain.

The apparent preservation of theta power in our data is noteworthy, particularly given that we observed axonal pathology of all major inputs to the hippocampus, including into the SL-M and dentate molecular layer, which receive major inputs that drive theta oscillations (Buzsáki, 2002). This apparent discrepancy could reflect a post-TBI compensatory mechanism whereby remaining intact inputs have "retuned" to maintain normal levels of synaptic activity. This may suffice to preserve observed anesthesia theta during low theta states, such as deep isoflurane anesthesia (Lustig et al., 2016), but could manifest as reduced theta power during high-theta states, such as exploratory behavior. Our findings are therefore not inconsistent with previous reports and underscore that others' observations of theta power loss after TBI are likely state dependent (Fedor et al., 2010; Lee et al., 2013, 2015). Variations in impact size and location on the skull, rodent species, and severity of injury reported in the FPI literature may also explain the variation in findings. Further, isoflurane itself can reduce neuronal firing rates and oscillatory power as well as shift the balance between inhibitory and excitatory current flow. We chose to perform this experiment under isoflurane anesthesia to facilitate the simultaneous recording of single-unit activity in both CA1 and MS. Therefore, our findings of specific power losses, firing pattern changes, or inhibitory activity under a constant anesthetic state require confirmation in the awake animal during the appropriate behavior. Nonetheless, alterations in coupling between coordinated synaptic inputs, as reflected in LFP oscillatory activity and local neuronal firing patterns, can provide important insights into the hippocampal network alterations that occur after TBI.

\section{Local CA1 neurons maintain normal firing rates, but cannot synchronize properly to stratum radiatum input after TBI}

Coordinated synaptic input into SR from CA3 plays a major role in organizing local CA1 pyramidal cell and interneuron firing patterns during states of consummatory behavior and non-REM sleep, and serves as a possible mechanism of local information encoding and distribution to efferent structures (Carr et al., 2011; Buzsáki and Moser, 2013). One might expect decreased individual CA1 neuron activity in the face of a reduction in the dominant oscillatory input after TBI, yet we found no changes in mean firing rates of isolated CA1 neurons, consistent with previous reports (Eakin and Miller, 2012; Munyon et al., 2014). However, we did find that isolated CA1 pyramidal cells could no longer synchronize properly to this dominant CA3 input. This is particularly significant as states dominated by CA3-derived sharp waves are the most synchronous states in the intact hippocampus, with the highest firing probability of CA1 neurons occurring during the negative peak of the sharp wave observed in the CA1 LFP (Buzsáki, 2015). If local CA1 neurons are unable to appropriately respond to a major input into SR, their ability to follow more complex oscillatory patterns, also critical for memory encoding and decoding, may be disrupted.

We suggest that since CA1 neurons were able to maintain normal firing rates, yet unable to synchronize appropriately to oscillatory SR inputs, a compensatory mechanism may have been able to adjust for firing rate. Whether intrinsic or synaptic, this may come at the cost of maintaining more subtle input/output relationships. If this is a homeostatic plasticity mechanism whereby the remaining inputs are strengthened to maintain consistent output, this may in turn lead to a loss 
of the ability of neurons to synchronize to their previously dominant input and affect normal computation in the dendritic arbor, such as ensemble organization within a sharp-wave ripple event (see below). Consistent with this idea, we have previously reported a mismatch between lost fiber volleys in the Schaffer collateral pathway and decreased CA1 EPSPs 1 week post-TBI in a largeanimal model, leading us to speculate that a form of homeostatic plasticity may be responsible (Turrigiano, 1999; Dinocourt et al., 2011; Wolf et al., 2017).

\section{Local CA1 high-frequency oscillations are decoupled from stratum radiatum input while synchronizing local neuron firing to a broader, nonspecific frequency range after TBI}

In addition to individual CA1 neuron action potentials, we also observed discrete high-frequency oscillatory events in the pyramidal cell layer that were coupled to the negative peak of the observed $\sim 10-15 \mathrm{~Hz}$ oscillatory input into SR. CA3-derived sharp-wave input into SR drives the occurrence of brief, high-frequency oscillations such as fast gamma and ripple oscillations in the pyramidal cell layer via local interactions between pyramidal cells and interneurons, resulting in a temporal coupling of sharp waves and these high-frequency events (Ylinen et al., 1995; Klausberger and Somogyi, 2008). SPW-Rs are known to organize ensembles of CA1 pyramidal cells into a particular firing sequence that recapitulates previous experiences, or future actions, and as such are thought to be critical for hippocampal-dependent memory (Carr et al., 2011). Although poorly defined under anesthesia, the observed perisomatic high-frequency oscillations in this study had frequencies that encompassed and extended beyond those expected of true ripples. Whether derived from similar mechanisms or not, the high-frequency oscillations observed in this study may represent both CA1 synchronized multiunit activity and local pyramidal cellinterneuronal interactions (Schomburg et al., 2012). We observed a power loss after injury within the extended ripple frequency range $(190-280 \mathrm{~Hz})$ in the pyramidal cell layer. We also observed a phase-amplitude decoupling of these high-frequency oscillations with the observed lowfrequency sharp wave-like $(\sim 10-15 \mathrm{~Hz})$ SR synaptic input, possibly representing rhythmic input from CA3 (Lustig et al., 2016). To the extent that discrete high-frequency oscillation events under anesthesia are generated by the same or similar mechanisms as true ripples, the observed decoupling and power losses after injury could reflect impaired pyramidal cell-interneuron interactions. Loss of coordinated CA3 inputs could also directly contribute to these findings, whether because of the observed Schaffer collateral pathology or CA3 neuronal injury, as reduced numbers of synchronized bursting CA3 pyramidal cells have been shown to result in fewer SPW$\mathrm{R}$ events and reduced ripple power in CA1 (Csicsvari et al., 2000).

In addition to synchronizing with oscillatory synaptic input, local CA1 neurons also synchronize to the phase of ripple oscillations (Buzsáki, 2015). Alternatively, fast oscillations $(>200 \mathrm{~Hz})$ may be due directly to synchronized local action potentials (Schomburg et al., 2012). It is therefore not surprising that we observed entrainment of putative CA1 pyramidal cells to high frequencies with a peak at $225 \mathrm{~Hz}$ in sham animals. Following TBI, the range of entrainment broadened with significant increases in entrainment below $(71-176 \mathrm{~Hz})$ and above $(>280 \mathrm{~Hz})$ the classic ripple frequency range. One explanation could be that local pyramidal cell-interneuron interactions responsible for ripple generation are impaired, leading to a "detuning" of pyramidal cell high-frequency entrainment within preferred ripple frequencies and allowing other mechanisms of fast oscillation generation to dominate. These may then entrain neurons across a wider and potentially inappropriate frequency range, such as high gamma oscillations, pathologic high-frequency oscillations, or default to current directly from pyramidal cell action potentials (Bragin et al., 1999; Sullivan et al., 2011; Schomburg et al., 2012). These results imply an impaired ability to maintain context-specific CA1 neuron ensemble organization after injury. However, the loss or dysfunction of SPW-R complexes in the awake behaving animal remains to be demonstrated.

\section{TBI leads to impaired hippocampal inhibitory modulation of the medial septum}

Generation and maintenance of hippocampal theta requires rhythmic input from the MS (Buzsáki, 2002). Experimental loss of theta power along with spatial memory performance can be rescued by MS stimulation (McNaughton et al., 2006b). However, the functional coupling between these structures is both reciprocal and state dependent (Dragoi et al., 1999; Borhegyi et al., 2004). For example, the majority of GABAergic MS neurons alter their response to synaptic inputs depending on the current hippocampal state (Toth et al., 1993; Dragoi et al., 1999; Borhegyi et al., 2004).

We found no evidence to support direct injury to the MS in the number of isolated MS cells, histologic inspection, or a reduction in overall firing rate. However, our histologic examinations were performed $7 \mathrm{~d}$ postinjury using markers that only detect cells or axons that are actively within the process of degeneration. As such, analyses will not identify cells or axons that may have degenerated acutely in the preceding days, the loss of which may influence electrophysiological findings.

After injury, we found intact coupling between MS neurons and CA1 anesthesia theta, but a loss of coordinated modulation of MS cells by the dominant CA $110-15 \mathrm{~Hz}$ oscillation in SR. This loss of MS neuron modulation appears to be a loss of inhibition and is coupled with both an increase in firing rate and an increased number of isolated GABAergic/glutamatergic MS neurons. As identification of putative single neurons from extracellular recordings requires adequate numbers of spontaneous action potentials (Thompson and Best, 1989), the detection of more MS neurons after injury likely reflects the overall increase in firing rate after injury rather than a true increase in the number of neurons postinjury. This finding may reflect loss of GABAergic feedback from CA1 stratum oriens interneurons to the MS (Müller and Remy, 2018). 
Disruption of HC modulation of the MS after TBI could reflect downstream consequences of the observed loss of synaptic activity into CA1 after injury, injury/dysfunction of CA1 interneurons, synaptic changes within the MS, acute loss of MS neurons that have already been cleared (see Discussion above), or a combination of these effects. Given the reciprocal relationship between the structures, our findings have implications for other $\mathrm{HC}$ states, including the proper generation and maintenance of theta during exploratory behavior.

In summary, we have provided the first in vivo evidence of anatomic layer-specific and frequency-specific changes in hippocampal oscillatory power $7 \mathrm{~d}$ after $\mathrm{TBI}$, reflecting both losses of coordinated synaptic input into CA1, as well as a loss of coordinated local CA1 neuronal activity. These findings provide one possible link between previous reports of CA1 power loss and the existing substantial structural and ex vivo electrophysiological evidence of CA3 neuronal loss as well as disrupted CA3-CA1 interactions after FPI, the most well established rodent model of TBI. Further, these findings provide the first in vivo evidence of (1) the failure of the principle output neurons of the hippocampus to properly synchronize to their synaptic inputs after TBI, a mechanism fundamental to hippocampal memory encoding and decoding; and (2) the failure of the hippocampus to exert normal modulation of an external structure, the medial septum, which may have implications for the proper state-dependent maintenance of hippocampal theta oscillations. We have found that the firing rates of CA1 neurons under isoflurane anesthesia appear unchanged, further supporting the hypothesis that the prominent dysfunction of the remaining intact network is in the ability to coordinate inputs and oscillations normally bound together that organize activity in the postsynaptic neurons. If confirmed in the behaving animal, these data could suggest that the normal SPW-R mechanism arising from CA3-CA1 interactions might be disrupted following TBI or neurons may not organize correctly in response. The ability to consolidate memory episodes during both waking and sleeping, as well as potentially distribute them beyond the hippocampus, may therefore be impaired. This may also explain the inability of the rats to recall the learned position of the platform in the water maze following injury. Finally, the preserved capacity of the remaining CA1 neurons to achieve normal firing rates $7 \mathrm{~d}$ post-TBI implies that therapeutic modulation of these networks may still allow for proper encoding of spatial and other cognitive information in the hippocampus, potentially using closed-loop strategies that are responsive to the current network state (Heck et al., 2014; Ezzyat et al., 2017).

\section{References}

Almeida-Suhett CP, Prager EM, Pidoplichko V, Figueiredo TH, Marini AM, Li Z, Eiden LE, Braga MFM (2015) GABAergic interneuronal loss and reduced inhibitory synaptic transmission in the hippocampal CA1 region after mild traumatic brain injury. Exp Neurol 273:11-23.

Bakshi A, Keck CA, Koshkin VS, LeBold DG, Siman R, Snyder EY, Mclntosh TK (2005) Caspase-mediated cell death predominates following engraftment of neural progenitor cells into traumatically injured rat brain. Brain Res 1065:8-19.
Baldwin SA, Gibson T, Callihan CT, Sullivan PG, Palmer E, Scheff SW (1997) Neuronal cell loss in the CA3 subfield of the hippocampus following cortical contusion utilizing the optical disector method for cell counting. J Neurotrauma 14:385-398.

Berens P (2009) CircStat: a MATLAB toolbox for circular statistics. J Stat Soft. Advance online publication. Retrieved Sep 23, 2009. doi:10.18637/jss.v031.i10.

Blumbergs PC, Scott G, Vis JM, Wainwright H, Simpson DA, McLean AJ (1995) Topography of axonal injury as defined by amyloid precursor protein and the sector scoring method in mild and severe closed head injury. J Neurotrauma 12:565-572.

Borhegyi Z, Varga V, Szilágyi N, Fabo D, Freund TF (2004) Phase segregation of medial septal GABAergic neurons during hippocampal theta activity. J Neurosci 24:8470-8479.

Bragin A, Engel J, Wilson CL, Fried I, Mathern GW (1999) Hippocampal and entorhinal cortex high-frequency oscillations $(100-500 \mathrm{~Hz})$ in human epileptic brain and in kainic acid-treated rats with chronic seizures. Epilepsia 40:127-137.

Broussard JI, Redell JB, Zhao J, Maynard ME, Kobori N, Perez Al, Hood KN, Zhang XO, Moore AN, Dash PK (2020) Mild traumatic brain injury decreases spatial information content and reduces place field stability of hippocampal CA1 neurons. J Neurotrauma 37:227-235.

Buzsáki G (1986) Hippocampal sharp waves: their origin and significance. Brain Res 398:242-252.

Buzsáki G (2002) Theta oscillations in the hippocampus. Neuron 33:325-340.

Buzsáki G (2015) Hippocampal sharp wave-ripple: a cognitive biomarker for episodic memory and planning. Hippocampus 25:10731188.

Buzsáki G, Moser El (2013) Memory, navigation and theta rhythm in the hippocampal-entorhinal system. Nat Neurosci 16:130-138.

Capruso DX, Levin HS (1992) Cognitive impairment following closed head injury. Neurol Clin 10:879-893.

Carr MF, Jadhav SP, Frank LM (2011) Hippocampal replay in the awake state: a potential substrate for memory consolidation and retrieval. Nat Neurosci 14:147-153.

Chen Z, Kontonotas D, Friedmann D, Pitts-Kiefer A, Frederick JR, Siman R, Neumar RW (2005) Developmental status of neurons selectively vulnerable to rapidly triggered post-ischemic caspase activation. Neurosci Lett 376:166-170.

Csicsvari J, Hirase H, Czurkó A, Mamiya A, Buzsáki G (1999) Fast network oscillations in the hippocampal CA1 region of the behaving rat. J Neurosci 19:RC20.

Csicsvari J, Hirase H, Mamiya A, Buzsáki G (2000) Ensemble patterns of hippocampal CA3-CA1 neurons during sharp wave-associated population events. Neuron 28:585-594.

Dinocourt C, Aungst S, Yang K, Thompson SM (2011) Homeostatic increase in excitability in area CA1 after Schaffer collateral transection in vivo. Epilepsia 52:1656-1665.

Dragoi G, Carpi D, Recce M, Csicsvari J, Buzsáki G (1999) Interactions between hippocampus and medial septum during sharp waves and theta oscillation in the behaving rat. $J$ Neurosci 19:6191-6199.

Eakin K, Miller JP (2012) Mild traumatic brain injury is associated with impaired hippocampal spatiotemporal representation in the absence of histological changes. J Neurotrauma 29:1180-1187.

Ezzyat Y, Kragel JE, Burke JF, Levy DF, Lyalenko A, Wanda P, O'Sullivan L, Hurley KB, Busygin S, Pedisich I, Sperling MR, Worrell GA, Kucewicz MT, Davis KA, Lucas TH, Inman CS, Lega BC, Jobst BC, Sheth SA, Zaghloul K, et al. (2017) Direct brain stimulation modulates encoding states and memory performance in humans. Curr Biol 27:1251-1258.

Fedor M, Berman RF, Muizelaar JP, Lyeth BG (2010) Hippocampal $\theta$ dysfunction after lateral fluid percussion injury. J Neurotrauma 27:1605-1615

Foster DJ, Wilson MA (2006) Reverse replay of behavioural sequences in hippocampal place cells during the awake state. Nature 440:680-683. 
Gentleman SM, Nash MJ, Sweeting CJ, Graham DI, Roberts GW (1993) Beta-amyloid precursor protein (beta APP) as a marker for axonal injury after head injury. Neurosci Lett 160:139-144.

Grady MS, Charleston JS, Maris D, Witgen BM, Lifshitz J (2003) Neuronal and glial cell number in the hippocampus after experimental traumatic brain injury: analysis by stereological estimation. J Neurotrauma 20:929-941.

Harris KD, Hirase H, Leinekugel X, Henze DA, Buzsáki G (2001) Temporal interaction between single spikes and complex spike bursts in hippocampal pyramidal cells. Neuron 32:141-149.

Harris KD, Csicsvari J, Hirase H, Dragoi G, Buzsáki G (2003) Organization of cell assemblies in the hippocampus. Nature 424:552-556.

Hazan L, Zugaro M, Buzsáki G (2006) Klusters, NeuroScope, NDManager: a free software suite for neurophysiological data processing and visualization. J Neurosci Methods 155:207-216.

Heck CN, King-Stephens D, Massey AD, Nair DR, Jobst BC, Barkley GL, Salanova V, Cole AJ, Smith MC, Gwinn RP, Skidmore C, Van Ness PC, Bergey GK, Park YD, Miller I, Geller E, Rutecki PA, Zimmerman R, Spencer DC, Goldman A, et al. (2014) Two-year seizure reduction in adults with medically intractable partial onset epilepsy treated with responsive neurostimulation: final results of the RNS System Pivotal trial. Epilepsia 55:432-441.

Hicks RR, Smith DH, Lowenstein DH, Marie RS, Mclntosh TK (1993) Mild experimental brain injury in the rat induces cognitive deficits associated with regional neuronal loss in the hippocampus. $J$ Neurotrauma 10:405-414.

Hoge CW, McGurk D, Thomas JL, Cox AL, Engel CC, Castro CA (2008) Mild traumatic brain injury in U.S. soldiers returning from Iraq. N Engl J Med 358:453-463.

Howard AL, Neu A, Morgan RJ, Echegoyen JC, Soltesz I (2007) Opposing modifications in intrinsic currents and synaptic inputs in post-traumatic mossy cells: evidence for single-cell homeostasis in a hyperexcitable network. J Neurophysiol 97:2394-2409.

Jia X, Kohn A (2011) Gamma rhythms in the brain. PLoS Biol 9: e1001045.

Johnson VE, Stewart JE, Begbie FD, Trojanowski JQ, Smith DH, Stewart W (2013a) Inflammation and white matter degeneration persist for years after a single traumatic brain injury. Brain 136:2842.

Johnson VE, Stewart W, Smith DH (2013b) Axonal pathology in traumatic brain injury. Exp Neurol 246:35-43.

Johnson VE, Stewart W, Weber MT, Cullen DK, Siman R, Smith DH (2016) SNTF immunostaining reveals previously undetected axonal pathology in traumatic brain injury. Acta Neuropathol 131:115135.

Johnson VE, Weber MT, Xiao R, Cullen DK, Meaney DF, Stewart W, Smith DH (2018) Mechanical disruption of the blood-brain barrier following experimental concussion. Acta Neuropathol 135:711726.

King C, Recce M, O'Keefe J (1998) The rhythmicity of cells of the medial septum/diagonal band of Broca in the awake freely moving rat: relationships with behaviour and hippocampal theta. Eur $\mathrm{J}$ Neurosci 10:464-477.

Klausberger T, Somogyi P (2008) Neuronal diversity and temporal dynamics: the unity of hippocampal circuit operations. Science 321:53-57.

Lee DJ, Gurkoff GG, Izadi A, Berman RF, Ekstrom AD, Muizelaar JP, Lyeth BG, Shahlaie K (2013) Medial septal nucleus theta frequency deep brain stimulation improves spatial working memory after traumatic brain injury. J Neurotrauma 30:131-139.

Lee DJ, Gurkoff GG, Izadi A, Seidl SE, Echeverri A, Melnik M, Berman RF, Ekstrom AD, Muizelaar JP, Lyeth BG, Shahlaie K (2015) Septohippocampal neuromodulation improves cognition after traumatic brain injury. J Neurotrauma 32:1822-1832.

Lisman J, Buzsáki G (2008) A neural coding scheme formed by the combined function of gamma and theta oscillations. Schizophr Bull 34:974-980.

Lustig B, Wang Y, Pastalkova E (2016) Oscillatory patterns in hippocampus under light and deep isoflurane anesthesia closely mirror prominent brain states in awake animals. Hippocampus 26:102109.

Maas AIR, Menon DK, Adelson PD, Andelic N, Bell MJ, Belli A, Bragge $P$, Brazinova A, Büki A, Chesnut RM, Citerio G, Coburn M, Cooper DJ, Crowder AT, Czeiter E, Czosnyka M, Diaz-Arrastia R, Dreier JP, Duhaime A-C, Ercole A, et al. (2017) Traumatic brain injury: integrated approaches to improve prevention, clinical care, and research. Lancet Neurol 16:987-1048.

Matthews RT, Lee WL (1991) A comparison of extracellular and intracellular recordings from medial septum/diagonal band neurons in vitro. Neuroscience 42:451-462.

McNaughton BL, Battaglia FP, Jensen O, Moser El, Moser MB (2006a) Path integration and the neural basis of the "cognitive map". Nat Rev Neurosci 7:663-678.

McNaughton N, Ruan M, Woodnorth MA (2006b) Restoring thetalike rhythmicity in rats restores initial learning in the Morris water maze. Hippocampus 16:1102-1110.

Mitra P, Bokil H (2008) Observed brain dynamics. Oxford, New York: Oxford UP.

Mitzdorf U (1985) Current Source-Density method and application in cat cerebral cortex: investigation of evoked potentials and EEg phenomena. Physiol Rev 65:37-100.

Monti JM, Voss MW, Pence A, McAuley E, Kramer AF, Cohen NJ (2013) History of mild traumatic brain injury is associated with deficits in relational memory, reduced hippocampal volume, and less neural activity later in life. Front Aging Neurosci 5:41.

Müller C, Remy S (2018) Septo-hippocampal interaction. Cell Tissue Res 373:565-575.

Munyon C, Eakin KC, Sweet JA, Miller JP (2014) Decreased bursting and novel object-specific cell firing in the hippocampus after mild traumatic brain injury. Brain research 1582:220-226.

Norris CM, Scheff SW (2009) Recovery of afferent function and synaptic strength in hippocampal CA1 following traumatic brain injury. J Neurotrauma 26:2269-2278.

O'Keefe J, Recce ML (1993) Phase relationship between hippocampal place units and the EEG theta rhythm. Hippocampus 3:317330.

Oo TF, Siman R, Burke RE (2002) Distinct nuclear and cytoplasmic localization of caspase cleavage products in two models of induced apoptotic death in dopamine neurons of the substantia nigra. Exp Neurol 175:1-9.

Paterno R, Metheny H, Xiong G, Elkind J, Cohen AS (2016) Mild traumatic brain injury decreases broadband power in area CA1. J Neurotrauma 33:1645-1649.

Paxinos G, Watson CRR (2007) The rat brain in stereotaxic coordinates, Ed 6. London, UK: Academic, Elsevier.

Pettersen KH, Devor A, Ulbert I, Dale AM, Einevoll GT (2006) Currentsource density estimation based on inversion of electrostatic forward solution: effects of finite extent of neuronal activity and conductivity discontinuities. J Neurosci Methods 154:116-133.

Pierce J, Smith DH, Trojanowski JQ, Mclntosh TK (1998) Enduring cognitive, neurobehavioral and histopathological changes persist for up to one year following severe experimental brain injury in rats. Neuroscience 87:359-369.

Reeves TM, Lyeth BG, Phillips LL, Hamm RJ, Povlishock JT (1997) The effects of traumatic brain injury on inhibition in the hippocampus and dentate gyrus. Brain Res 757:119-132.

Rossant C, Kadir SN, Goodman DFM, Schulman J, Hunter MLD, Saleem AB, Grosmark A, Belluscio M, Denfield GH, Ecker AS, Tolias AS, Solomon S, Buzsáki G, Carandini M, Harris KD (2016) Spike sorting for large, dense electrode arrays. Nat Neurosci 19:634-641.

Santhakumar V, Ratzliff AD, Jeng J, Toth Z, Soltesz I (2001) Longterm hyperexcitability in the hippocampus after experimental head trauma. Ann Neurol 50:708-717.

Scheff SW, Price DA, Hicks RR, Baldwin SA, Robinson S, Brackney C (2005) Synaptogenesis in the hippocampal CA1 field following traumatic brain injury. J Neurotrauma 22:719-732. 
Schmued LC, Stowers CC, Scallet AC, Xu L (2005) Fluoro-Jade C results in ultra high resolution and contrast labeling of degenerating neurons. Brain Res 1035:24-31.

Schomburg EW, Anastassiou CA, Buzsáki G, Koch C (2012) The spiking component of oscillatory extracellular potentials in the rat hippocampus. J Neurosci 32:11798-11811.

Smith DH, Okiyama K, Thomas MJ, Claussen B, McIntosh TK (1991) Evaluation of memory dysfunction following experimental brain injury using the Morris water maze. J Neurotrauma 8:259-269.

Smith DH, Chen X-H, Pierce JES, Wolf JA, Trojanowski JQ, Graham DI, Mclntosh TK (1997) Progressive atrophy and neuron death for one year following brain trauma in the rat. J Neurotrauma 14:715727.

Sullivan D, Csicsvari J, Mizuseki K, Montgomery S, Diba K, Buzsaki G (2011) Relationships between hippocampal sharp waves, ripples, and fast gamma oscillation: influence of dentate and entorhinal cortical activity. J Neurosci 31:8605-8616.

Thompson LT, Best PJ (1989) Place cells and silent cells in the hippocampus of freely-behaving rats. J Neurosci 9:2382-2390.

Tort ABL, Komorowski R, Eichenbaum H, Kopell N (2010) Measuring phase-amplitude coupling between neuronal oscillations of different frequencies. J Neurophysiol 104:1195-1210.

Toth K, Borhegyi Z, Freund TF (1993) Postsynaptic targets of GABAergic hippocampal neurons in the medial septum-diagonal band of Broca complex. J Neurosci 13:3712-3724.
Toth Z, Hollrigel GS, Gorcs T, Soltesz I (1997) Instantaneous perturbation of dentate interneuronal networks by a pressure wave-transient delivered to the neocortex. J Neurosci 17:8106-8117.

Turrigiano GG (1999) Homeostatic plasticity in neuronal networks: the more things change, the more they stay the same. Trends Neurosci 22:221-227.

Vorhees CV, Williams MT (2006) Morris water maze: procedures for assessing spatial and related forms of learning and memory. Nat Protoc 1:848-858.

Weber MT, Arena JD, Xiao R, Wolf JA, Johnson VE (2019) Clarity reveals a more protracted temporal course of axon swelling and disconnection than previously described following traumatic brain injury. Brain Pathol 29:437-450.

Wilson L, Stewart W, Dams-O'Connor K, Diaz-Arrastia R, Horton L, Menon DK, Polinder S (2017) The chronic and evolving neurological consequences of traumatic brain injury. Lancet Neurol 16:813-825.

Wolf JA, Koch PF (2016) Disruption of network synchrony and cognitive dysfunction after traumatic brain injury. Front Syst Neurosci 10:43.

Wolf JA, Johnson BN, Johnson VE, Putt ME, Browne KD, Mietus CJ, Brown DP, Wofford KL, Smith DH, Grady MS, Cohen AS, Cullen DK (2017) Concussion induces hippocampal circuitry disruption in swine. J Neurotrauma 34:2303-2314.

Ylinen A, Bragin A, Nadasdy Z, Jando G, Szabo I, Sik A, Buzsaki G (1995) Sharp wave-associated high-frequency oscillation $(200 \mathrm{~Hz})$ in the intact hippocampus: network and intracellular mechanisms. J Neurosci 15:30-46. 